TRANSACTIONS OF THE

AMERICAN MATHEMATICAL SOCIETY

Volume 350, Number 9, September 1998, Pages 3767-3796

S 0002-9947(98)01980-1

\title{
TESSELLATIONS OF SOLVMANIFOLDS
}

\author{
DAVE WITTE
}

\begin{abstract}
Let $A$ be a closed subgroup of a connected, solvable Lie group $G$, such that the homogeneous space $A \backslash G$ is simply connected. As a special case of a theorem of C. T. C. Wall, it is known that every tessellation $A \backslash G / \Gamma$ of $A \backslash G$ is finitely covered by a compact homogeneous space $G^{\prime} / \Gamma^{\prime}$. We prove that the covering map can be taken to be very well behaved - a "crossed" affine map. This establishes a connection between the geometry of the tessellation and the geometry of the homogeneous space. In particular, we see that every geometrically-defined flow on $A \backslash G / \Gamma$ that has a dense orbit is covered by a natural flow on $G^{\prime} / \Gamma^{\prime}$.
\end{abstract}

\section{INTRODUCTION}

Let $G$ be a transitive group of diffeomorphisms of a manifold $M$. (For our purposes, the most important case is when $M=\mathbb{R}^{n}$.) The choice of a particular group $G$ endows $M$ with the structure of a homogeneous space. (In other words, the choice of a particular group $G$ represents the choice of a particular geometric structure on $M$, in the sense of Klein's Erlangen Program.) We always assume that $G$ is a finite-dimensional Lie group, and that $G$ is connected (or, at the worst, that $G$ has only finitely many connected components). It is well known that $M$ is $G$-equivariantly diffeomorphic to a coset space $A \backslash G$ (or to $G / A$, if one prefers to have $G$ act on the left) [V, Lem. 2.9.2, p. 76].

In this paper, $G$ is usually solvable, in which case the pair $(M, G)$ is said to be a solvmanifold. More simply, we usually refer to $M$ as a solvmanifold, with the choice of a particular solvable group $G$ being understood.

Now assume $M=\mathbb{R}^{n}$. If $\Gamma$ is a properly discontinuous subgroup of $G$, such that $\mathbb{R}^{n} / \Gamma$ is compact, then we may refer to $\mathbb{R}^{n} / \Gamma$ as a tessellation, because the $\Gamma$-translates of a fundamental domain tessellate $\mathbb{R}^{n}$. If $G$ is solvable, then $\mathbb{R}^{n} / \Gamma$ is said to be a solvtessellation. Note that a tessellation $\mathbb{R}^{n} / \Gamma$ is a manifold if $\Gamma$ acts freely on $\mathbb{R}^{n}$. Because $\Gamma$ has a torsion-free subgroup of finite index $[R$, Lem. 4.6, p. 57], it follows that every solvtessellation has a finite branched cover that is a manifold. (Hence, every tessellation is an "orbifold" [M, Appendix A].)

One may study tessellations of solvmanifolds other than $\mathbb{R}^{n}$, but the most important case is where the solvmanifold is simply connected. Then, because every simply connected solvmanifold is diffeomorphic to $\mathbb{R}^{n}$ (cf. [M1, Prop. 11.2]), there is no real loss in restricting attention to $\mathbb{R}^{n}$.

Received by the editors October 6, 1994 and, in revised form, November 5, 1996.

1991 Mathematics Subject Classification. Primary 22E25, 22E40, 53C30; Secondary 05B45, 20G20, 20H15, 57S20, 57S30. 
The homeomorphism types of solvtessellations are understood (modulo finite covers), because of fundamental work of C. T. C. Wall [Wa, $\S 15 \mathrm{~B}]$ on aspherical manifolds with polycyclic fundamental group. Namely, Wall's work implies that some finite (branched) cover of $\mathbb{R}^{n} / \Gamma$ is homeomorphic to a homogeneous space (more precisely, a solvmanifold). (The lecture notes of Farrell and Jones [FJ] provide a survey of related work in surgery theory.) In other words, there is a finite group $F$ of diffeomorphisms of some solvmanifold $G^{\prime} / \Gamma^{\prime}$, such that $\mathbb{R}^{n} / \Gamma \approx$ $\left(G^{\prime} / \Gamma^{\prime}\right) / F$. If $F$ acts freely, this means that $\mathbb{R}^{n} / \Gamma$ is an "infrasolvmanifold."

Theorem 1.1 (Wall, cf. [Wa, §15B] or [FJ, Theorem 2.16]). Let $\Gamma$ be a properly discontinuous subgroup of a connected, solvable Lie group $G$ that acts transitively on $\mathbb{R}^{n}$. If $\mathbb{R}^{n} / \Gamma$ is compact, then there is some discrete subgroup $\Gamma^{\prime}$ of some simply connected, solvable Lie group $G^{\prime}$, such that some finite branched cover of $\mathbb{R}^{n} / \Gamma$ is homeomorphic to $G^{\prime} / \Gamma^{\prime}$.

Another way of stating the theorem is that, to construct a representative of every homeomorphism class of solvtessellations of $\mathbb{R}^{n}$, modulo finite covers, there is no need to consider groups $G$ of any dimension other than $n$. Furthermore, the representation of $G$ as a group of diffeomorphisms of $\mathbb{R}^{n}$ can be taken to be the action of $G$ on itself by right multiplication.

The assumption that $G$ is solvable cannot be eliminated from the theorem. For example, to construct a compact $n$-manifold of constant negative curvature, one takes $G$ to be the group of orientation-preserving isometries of hyperbolic $n$ space $\mathbb{H}^{n}$ (that is, $G \cong \mathrm{SO}(1, n)$ ). The dimension of $G$ is then $n(n+1) / 2$, which is much larger than $n$.

Because Wall's proof is topological, it does not seem to show any connection between the geometry of $\mathbb{R}^{n} / \Gamma$ and that of $G^{\prime} / \Gamma^{\prime}$. (Indeed, Wall's statement of the theorem applies in a much more general setting, where the manifolds involved have no a priori geometric structure.) In the present paper, we give an algebraic proof of the theorem, thus exhibiting the precise relationship between the geometry of the two spaces.

Ideally, one might hope that some covering map $\pi$ from $G^{\prime} / \Gamma^{\prime}$ to $\mathbb{R}^{n} / \Gamma$ would respect the geometry, in the sense that every local isometry of $G^{\prime} / \Gamma^{\prime}$ would push down, via $\pi$, to a local isometry of $\mathbb{R}^{n} / \Gamma$. Since the geometry of $\mathbb{R}^{n} / \Gamma$ is represented by the group $G$, and the geometry of $G^{\prime} / \Gamma^{\prime}$ is represented by the group $G^{\prime}$, this can be restated more precisely by saying that, ideally, there would be a group homomorphism $\phi: G^{\prime} \rightarrow G$, such that

$$
(x g)^{\tilde{\pi}}=x^{\tilde{\pi}} \cdot g^{\phi}
$$

for every $x$ and $g$ in $G^{\prime}$, where $\tilde{\pi}$ is the lift of $\pi$ to a map $G^{\prime} \rightarrow \mathbb{R}^{n}$ of the universal covers. This is equivalent to saying that, ideally, $\pi$ would be an affine map.

Definition 1.2. For any choice of a basepoint $x_{0} \in \mathbb{R}^{n}$, the action of $G$ on $\mathbb{R}^{n}$ provides a continuous map $G \rightarrow \mathbb{R}^{n}: g \mapsto x_{0} g$. Then, if $\phi: G^{\prime} \rightarrow G$ is any group homomorphism, we have a composite map

$$
\phi^{\prime}: G^{\prime} \stackrel{\phi}{\longrightarrow} G \stackrel{g \mapsto x_{0} g}{\longrightarrow} \mathbb{R}^{n},
$$

which is an affine map. If, in addition, $\left(\Gamma^{\prime}\right)^{\phi} \subset \Gamma$, then $\phi^{\prime}$ induces a well-defined map $\phi^{\prime \prime}: G^{\prime} / \Gamma^{\prime} \rightarrow \mathbb{R}^{n} / \Gamma$, and $\phi^{\prime \prime}$ is also called an affine map.

Unfortunately, it is not always possible to arrange for the covering map $G^{\prime} / \Gamma^{\prime} \rightarrow$ $\mathbb{R}^{n} / \Gamma$ to be affine. (For example, Figure 4.1 shows a solvtessellation that is homeo- 
morphic to the ordinary 2-torus $\mathbb{T}^{2}$, but does not have the geometric structure of any solvmanifold.) Thus, in some cases, the covering map must distort the geometry. It turns out that the obstructions arise from certain "rotations" associated to $G$, that is, from certain elements in compact, abelian groups of automorphisms of $G$. To eliminate the obstructions, we add on more rotations to $G$, in order to cancel the ones that are causing the difficulty. Thus, instead of a homomorphism from $G^{\prime}$ into $G$, we deal with a homomorphism from $G^{\prime}$ into the semidirect product $T \ltimes G$, for some compact, abelian subgroup $T$ of Aut $G$. This leads to "crossed homomorphisms" and the corresponding "crossed affine maps."

Definition 1.3 (cf. [CE, p. 168]). Let $G$ and $G^{\prime}$ be groups. A function $\phi: G^{\prime} \rightarrow G$ is a crossed homomorphism if there is some homomorphism $\sigma: G^{\prime} \rightarrow$ Aut $G$, such that the function

$$
\sigma \times \phi: G^{\prime} \rightarrow \text { Aut } G \ltimes G: g \mapsto\left(g^{\sigma}, g^{\phi}\right)
$$

is a homomorphism. It is easy to check that $\sigma \times \phi$ is a homomorphism iff

$$
(g h)^{\phi}=\left(g^{\phi}\right)^{h^{\sigma}} h^{\phi}, \quad \forall g, h \in G^{\prime} .
$$

In particular, every homomorphism is a crossed homomorphism, with $\sigma$ being the trivial homomorphism $\left(h^{\sigma}=e\right)$. if

For a subgroup $\Gamma^{\prime}$ of $G^{\prime}$, we say that a crossed homomorphism $\phi$ is $\Gamma^{\prime}$-equivariant

$$
(g \gamma)^{\phi}=g^{\phi} \gamma^{\phi}
$$

for every $g \in G^{\prime}$ and $\gamma \in \Gamma^{\prime}$. In other words, the associated homomorphism $\sigma: G^{\prime} \rightarrow$ Aut $G$ is trivial on $\Gamma^{\prime}$, that is, $\left(\Gamma^{\prime}\right)^{\sigma}=e$. In particular, every homomorphism is $\Gamma^{\prime}$-equivariant, for every subgroup $\Gamma^{\prime}$.

We now generalize the definition of an affine map, by allowing crossed homomorphisms, instead of only allowing homomorphisms. The catch is that, to ensure that a map from $G^{\prime} / \Gamma^{\prime}$ to $\mathbb{R}^{n} / \Gamma$ is well defined, we assume $\Gamma^{\prime}$-equivariance.

Definition 1.4 (cf. Definition 1.3). For any choice of a basepoint $x_{0} \in \mathbb{R}^{n}$, the action of $G$ on $\mathbb{R}^{n}$ provides a continuous map $G \rightarrow \mathbb{R}^{n}: g \mapsto x_{0} g$. Then, if $\phi: G^{\prime} \rightarrow$ $G$ is any crossed homomorphism, we have a composite map

$$
\phi^{\prime}: G^{\prime} \stackrel{\phi}{\longrightarrow} G \stackrel{g \mapsto x_{0} g}{\longrightarrow} \mathbb{R}^{n}
$$

which is a crossed affine map. If, in addition, $\left(\Gamma^{\prime}\right)^{\phi} \subset \Gamma$, and $\phi$ is $\Gamma^{\prime}$-equivariant, then $\phi^{\prime}$ induces a well-defined map $\phi^{\prime \prime}: G^{\prime} / \Gamma^{\prime} \rightarrow \mathbb{R}^{n} / \Gamma$, and $\phi^{\prime \prime}$ is also called a crossed affine map.

Main Theorem 7.2'. Let $\Gamma$ be a properly discontinuous subgroup of a connected, transitive, solvable Lie group of diffeomorphisms of $\mathbb{R}^{n}$. If $\mathbb{R}^{n} / \Gamma$ is compact, then there is some discrete subgroup $\Gamma^{\prime}$ of some simply connected, solvable Lie group $G^{\prime}$, and a crossed affine map $\phi: G^{\prime} / \Gamma^{\prime} \rightarrow \mathbb{R}^{n} / \Gamma$, such that $\phi$ is a covering map, with finite fibers.

A more explicit description of the crossed affine map $\phi$ appears in Section 7.

Crossed affine maps are $C^{\infty}$. (In fact, they are real analytic [V, Thm. 2.11.2, p. 93].) Hence, we have the following corollary. 
Corollary 1.5. Let $\Gamma$ be a properly discontinuous subgroup of a connected, transitive, solvable Lie group of diffeomorphisms of $\mathbb{R}^{n}$. If $\mathbb{R}^{n} / \Gamma$ is compact, then there is some discrete subgroup $\Gamma^{\prime}$ of some simply connected, solvable Lie group $G^{\prime}$, such that some finite cover of $\mathbb{R}^{n} / \Gamma$ is real-analytically diffeomorphic to $G^{\prime} / \Gamma^{\prime}$.

G. D. Mostow [M1, Thm. A], [R, Thm. 3.6, p. 50] proved that compact solvmanifolds with isomorphic fundamental groups are diffeomorphic. (In fact, they are real-analytically diffeomorphic [W2].) Hence, we have the following corollary.

Corollary 1.6. Let $\Gamma$ and $\Gamma^{\prime}$ be properly discontinuous subgroups of connected, solvable Lie groups $G$ and $G^{\prime}$ that act transitively (and real analytically) on $\mathbb{R}^{n}$. Assume $\mathbb{R}^{n} / \Gamma$ and $\mathbb{R}^{n} / \Gamma^{\prime}$ are compact. If $\Gamma$ and $\Gamma^{\prime}$ are isomorphic, then some finite cover of $\mathbb{R}^{n} / \Gamma$ is (real analytically) diffeomorphic to a finite cover of $\mathbb{R}^{n} / \Gamma^{\prime}$.

Definition 1.7. The geometric structure of a homogeneous space can be used to construct natural dynamical systems [AGH]. Namely, each one-parameter subgroup of $G$ defines an action of $\mathbb{R}$ on the homogeneous space, which is to say that each one-parameter subgroup of $G$ defines a flow on the homogeneous space. If $G$ is solvable, this flow is called a solvflow. (In the general case, some authors call this a "G-induced" flow.)

The geometric structure of a tessellation $\mathbb{R}^{n} / \Gamma$ can also be used to construct natural dynamical systems, in a slightly different way. Let $v$ be a tangent vector to $\mathbb{R}^{n}$ at 0 . If $v$ is invariant under $G_{0}$, the isotropy group of 0 in $G$, then the $G$ translates of $v$ form a well-defined, $G$-invariant vector field $\chi$ on $\mathbb{R}^{n}$. (This means $\chi$ is invariant under the group that defines the geometric structure on $\mathbb{R}^{n}$, so we say that $\chi$ is a geometric vector field on $\mathbb{R}^{n}$.) Being $G$-invariant, the vector field $\chi$ is $\Gamma$-invariant, so $\chi$ pushes down to a well-defined vector field on the tessellation $\mathbb{R}^{n} / \Gamma$, and we call this a geometric vector field on $\mathbb{R}^{n} / \Gamma$. The flow generated by a geometric vector field is called a geometric flow on $\mathbb{R}^{n} / \Gamma$. It may be the case that 0 is the only $G_{0}$-invariant tangent vector at 0 , in which case this construction is not interesting, but there are important examples, such as the geodesic flow on the unit tangent bundle of a surface of constant negative curvature.

A diffeomorphism may transform a geometric flow into something terrible, but crossed affine maps, as in the Main Theorem, are well behaved. Thus, we have the following corollary.

Corollary 8.1'. If a geometric flow on a solvtessellation has a dense orbit, then the flow is finitely covered by a solvflow.

Therefore, the extensive theory of solvflows $[\mathrm{AGH}],[\mathrm{A} 1],[\mathrm{A} 2],[\mathrm{BM}]$ can be applied to the study of geometric flows on solvtessellations. For example, we have the following consequences.

Corollary 8.2'. If a geometric flow on a solvtessellation has no nonconstant, $C^{\infty}$, invariant functions, then the flow is ergodic, and is finitely covered by an ergodic solvflow.

Corollary $\mathbf{8 . 3}^{\prime}$. Every $L^{2}$ eigenfunction of any ergodic geometric flow on any solvtessellation is equal to a $C^{\infty}$ function (a.e.).

Corollary 8.4'. A geometric flow on a solvtessellation $\mathbb{R}^{n} / \Gamma$ is minimal (that is, every orbit is dense) if and only if (1) the flow has a dense orbit, and (2) the fundamental group $\Gamma$ is virtually nilpotent. 
The Main Theorem can be viewed as an analogue for tessellations of solvmanifolds of the following classical theorem of Bieberbach on tessellations of Euclidean space.

Theorem 1.8 (Bieberbach). Let $\Gamma$ be a properly discontinuous group of Euclidean isometries of $\mathbb{R}^{n}$. If $\mathbb{R}^{n} / \Gamma$ is compact, then some finite cover of $\mathbb{R}^{n} / \Gamma$ is isometric to a flat n-torus.

Bieberbach's conclusion is usually stated differently: there is a finite-index subgroup $\Gamma^{\prime}$ of $\Gamma$ that consists entirely of translations [C, Thm. I.3.1, p. 17]. This implies that $\Gamma^{\prime}$ is contained in the group $B$ of all translations, and $B / \Gamma^{\prime}$ is compact, so, in terms of the following definition, we may say that $\Gamma^{\prime}$ has a syndetic hull in $G$.

Definition 1.9 (cf. [FG, p. 6]). Let $\Gamma$ be a closed subgroup of a connected Lie group $G$. A syndetic hull of $\Gamma$ in $G$ is a connected subgroup $B$ of $G$ containing $\Gamma$, such that $B / \Gamma$ is compact.

The group of translations is simply transitive on $\mathbb{R}^{n}$, so we see that Bieberbach proved the following condition, in the case where $G$ is the group of Euclidean rigid motions.

Bieberbach Condition 1.10. If $\Gamma$ is a properly discontinuous subgroup of $G$, such that $\mathbb{R}^{n} / \Gamma$ is compact, then some finite-index subgroup $\Gamma^{\prime}$ of $\Gamma$ has a syndetic hull $B$, such that $B$ acts simply transitively on $\mathbb{R}^{n}$.

The Bieberbach Condition implies that $\mathbb{R}^{n} / \Gamma^{\prime}$ is diffeomorphic to the compact homogeneous space $B / \Gamma^{\prime}$ (see 2.3), so it implies the conclusion of the Main Theorem.

It is easy to prove the Bieberbach Condition if $G$ is nilpotent (see 3.8). (In fact, there is no need to pass to a finite-index subgroup of $\Gamma$ in this case.) Unfortunately, the Bieberbach Condition can fail for a general solvable group $G$, because there are examples where no syndetic hull exists (see Example 4.24 and Figure 4.1). (However, Proposition 6.1 shows that the syndetic hull is transitive, if it exists.) Therefore, in our proof of the Main Theorem, we replace $G$ with a related group $G^{\Delta}$ in which some finite-index subgroup $\Gamma^{\prime}$ does have a syndetic hull $B^{\Delta}$. (The construction of $G^{\Delta}$ is based on the "nilshadow" invented by L. Auslander and R. Tolimieri $[\mathrm{AT}]$.) The proof is then completed by showing that $\mathbb{R}^{n} / \Gamma^{\prime}$ is diffeomorphic to $B^{\Delta} / \Gamma^{\prime}$.

The outline of the paper is as follows.

$\S 2$. Preliminaries from Lie Theory

$\S 3$. Tessellations of nilmanifolds

$\S 4$. Background from the theory of real algebraic groups

$\S 5$. The nilshadow map

$\S 6$. The syndetic hull is transitive

$\S 7$. Proof of the main theorem

§8. Geometric flows on solvtessellations

Acknowledgments. I am grateful to Geoff Mess for calling my attention to the literature on aspherical manifolds with polycyclic fundamental groups, and for pointing out its close connection to the content of this paper. Also, I would like to thank Frank Morgan for interesting conversations that led to improvements in my results, and I am grateful to Robert Gutschera for several helpful references. This research was partially supported by a grant from the National Science Foundation. 


\section{Preliminaries from Lie Theory}

We assume familiarity with the basic theory of Lie groups, including the Lie algebra, the exponential map, the adjoint representation, semidirect products, and homogeneous spaces, as found in standard texts, such as [Ho] and [V].

Notation 2.1. A lower-case greek letter ( $\operatorname{such}$ as $\sigma, \pi, \tau$, or $\phi$ ) always denotes a function. An upper-case roman letter denotes a group or set. A lower-case roman letter denotes an element of a group, except that the letters from the middle of the alphabet $(i, j, k, l, m, n)$ denote integers. We often write functions as superscripts. For example, $x^{\sigma}$ means the same thing as $\sigma(x)$. Writing a group element as a superscript denotes conjugation, that is, $a^{b}=b^{-1} a b$.

The following basic lemma will be used to restate the Main Theorem, and other statements of the introduction, in a group-theoretic form that is more convenient for proofs.

Lemma 2.2 ([V, Lem. 2.9.2, p. 76]). Suppose $G$ is a transitive group of diffeomorphisms of $\mathbb{R}^{n}$, and let $A=G_{0}$ be the isotropy group of 0 in $G$. Then $\mathbb{R}^{n}$ is $G$ equivariantly diffeomorphic to $A \backslash G$.

If $G$ is simply transitive, then $G_{0}$ is trivial, so we have the following corollary.

Corollary 2.3. If $G$ is a simply transitive group of diffeomorphisms of $\mathbb{R}^{n}$, then $\mathbb{R}^{n}$ is $G$-equivariantly diffeomorphic to $G$. Therefore, for every subgroup $\Gamma$ of $G$, the quotient $\mathbb{R}^{n} / \Gamma$ is diffeomorphic to the homogeneous space $G / \Gamma$.

In the same spirit, the following result translates the definition of geometric flows into the more amenable language of group theory. It is based on the observation that multiplication on the left commutes with multiplication on the right.

Proposition 2.4. Let $A \backslash G / \Gamma$ be a tessellation. If $X$ is a connected Lie subgroup of $G$ that contains $A$ as a codimension-one, normal subgroup, then the action of $X / A$ on $A \backslash G / \Gamma$ is a (well-defined) geometric flow. Conversely, every geometric flow on $A \backslash G / \Gamma$ arises in this way.

We now recall some basic properties of maximal compact subgroups. Most of these results were proved by Iwasawa [I1], [I2].

Proposition 2.5 ([I1, Thm. 6], [Ho, Thm. XV.3.1, pp. 180-181]). Every compact subgroup of a connected Lie group $G$ is contained in a maximal compact subgroup, and all maximal compact subgroups of $G$ are conjugate.

Theorem 2.6 ([I1, Thm. 6], [Ho, Thm. XV.3.1, pp. 180-181]). If $K$ is a maximal compact subgroup of a connected Lie group $G$, then $G$ is homeomorphic to the cartesian product $K \times \mathbb{R}^{n}$, for some $n$. In particular, $K$ is connected, and $G$ is simply connected if and only if $K$ is simply connected.

Proposition 2.7 (cf. [Ho, Thm. XIII.1.3, p. 144]). If $G$ is a compact, connected, solvable Lie group, then $G$ is abelian. Hence, $G \cong \mathbb{T}^{n}$, for some $n$.

Corollary 2.8. A connected, solvable Lie group $G$ is simply connected iff $G$ has no nontrivial compact subgroups.

Definition 2.9 ([V, p. 244]). The nilradical of a Lie group $G$, denoted nil $G$, is the unique maximal connected, nilpotent, normal subgroup of $G$. 
Lemma 2.10 (cf. [I2, Satz 5]). If $G$ is a connected Lie group, then nil $G$ has a unique maximal compact subgroup $K$, and $K \subset Z(G)$. In other words, every compact subgroup of nil $G$ is normal (indeed, central) in $G$.

Lemma 2.11 ([W2, 2$])$. Let $A$ be a closed subgroup of a connected, solvable Lie group $G$. Then $A \backslash G$ is simply connected iff (1) A contains a maximal compact subgroup of $G$, and (2) $A$ is connected.

The following simple lemma is often used when we need to show that a subgroup $B$ acts transitively on a homogeneous space $A \backslash G$. It allows us to simplify the problem by replacing $G$ and $A$ with smaller subgroups $H$ and $A \cap H$.

Lemma 2.12. Let $A, B$, and $H$ be closed subgroups of a connected Lie group $G$. If $B \subset H$ and $A H=G$, then the natural inclusion of $(A \cap H) \backslash H$ in $A \backslash G$ is a $B$-equivariant homeomorphism. In particular:

1. $B$ is transitive on $(A \cap H) \backslash H$ iff $B$ is transitive on $A \backslash G$;

2. $(A \cap H) \backslash H / B$ is homeomorphic to $A \backslash G / B$;

3. $A B=G$ iff $(A \cap H) B=H$;

4. every double coset $A g B$ is closed iff every double coset $(A \cap H) h B$ is closed; and

5. $A \backslash G / B$ is compact iff $(A \cap H) \backslash H / B$ is compact.

Furthermore, if $A \backslash G$ is simply connected and $H$ is connected, then $A \cap H$ is connected.

We close the section by recalling three basic results on solvable groups.

Lemma 2.13 ([Ho, Thm. XII.2.2, p. 137]). Every connected subgroup of a simply connected, solvable Lie group $G$ is closed and simply connected.

Lemma 2.14 (cf. [V, Cor. 3.8.4, p. 207]). If $G$ is a connected, solvable Lie group, then $G / \operatorname{nil} G$ is abelian. In other words, $[G, G] \subset \operatorname{nil} G$.

Lemma 2.15. If $G$ is a simply connected Lie group, then nil $G$ is simply connected.

Proof. From [V, Thm. 3.18.13, p. 244], we see that nil $G$ is contained in a simply connected, solvable subgroup of $G$, so nil $G$ is simply connected (see 2.13).

\section{Tessellations of Nilmanifolds}

This section presents a proof of the Bieberbach Condition (1.10), the Main Theorem, and Corollary 8.1, in the case where $G$ is nilpotent. The proofs are quite short, and the statements of the results $(3.8,3.9$, and 3.11) do not require technicalities such as finite-index subgroups, finite covers, or crossed affine maps. We begin by showing that syndetic hulls are transitive (3.1 and 3.2), and perhaps simply transitive (3.4). We then note that a syndetic hull always exists (3.6 and 3.7), and deduce the Bieberbach Condition (3.8). The other results (3.9) and (3.11) are easy consequences.

Proposition 3.1. Let $A \backslash G$ be a simply connected homogeneous space of a connected, nilpotent Lie group $G$, and let $B$ be a connected subgroup of $G$, such that $A \backslash G / B$ is compact. Then $A B=G$.

Proof. We may assume $B$ is a proper subgroup of $G$. Because $G$ is nilpotent, this implies $B$ is a proper subgroup of $N_{G}(B)$ [Ha, Cor. 10.3.1, p. 154] and $N_{G}(B)$ is 
connected [Bou, Prop. III.16, p. 348]. Therefore, by induction on the codimension of $B$, we have $A \cdot N_{G}(B)=G$. Thus, by Lemma 2.12 , we may assume $G=N_{G}(B)$, which means $B$ is normal in $G$. Then $A B$ is a subgroup of $G$, and $(A B) \backslash G$ is simply connected, because $A \backslash G$ is simply connected and $B$ is connected (see 2.11). Therefore, $(A B) \backslash G$ is homeomorphic to $\mathbb{R}^{n}$, for some $n$ (cf. [M1, Prop. 11.2]). On the other hand, we know $(A B) \backslash G$ is compact, because $A \backslash G / B$ is compact. Therefore, $(A B) \backslash G$ is trivial, which means $A B=G$.

Corollary 3.2. Let $A$ and $B$ be connected Lie subgroups of a simply connected, nilpotent Lie group $G$. If $A \backslash G / B$ is compact, then $A B=G$.

Definition 3.3. A homogeneous space $A \backslash G$ of a Lie group $G$ is faithful if the action of $G$ on $A \backslash G$ is faithful or, equivalently, if $A$ contains no nontrivial, normal subgroup of $G$. Because any such normal subgroup could be modded out, there is usually no loss in assuming that a homogeneous space is faithful.

Theorem 3.4. Let $A \backslash G$ be a simply connected, faithful homogeneous space of a connected, nilpotent Lie group $G$, and let $\Gamma$ be a discrete subgroup of $G$ that acts properly on $A \backslash G$, such that $A \backslash G / \Gamma$ is compact. If $B$ is any syndetic hull of $\Gamma$ in $G$, then $A B=G$ and $A \cap B=e$. In other words, $B$ is simply transitive on $A \backslash G$.

Proof. Since $A \backslash G / \Gamma$ is compact, we know that $A \backslash G / B$ is compact. Because $B$ is connected, this implies $A B=G$ (see 3.1).

Because $\Gamma$ acts properly on $A \backslash G$, and $\Gamma$ is cocompact in $B$, we know $B$ acts properly on $A \backslash G$, so $A \cap B$ is compact. Therefore, $A \cap B \subset Z(G)$ (see 2.10), so $A \cap B \triangleleft G$. Because $A \backslash G$ is faithful, this implies $A \cap B=e$.

Remark 3.5. The conclusion of Theorem 3.4 can fail if $A \backslash G$ is not simply connected. For example, let $G$ be a nontrivial, compact, abelian group, and let $A=B=\Gamma=e$. For a more dramatic example, in which no syndetic hull of $\Gamma$ satisfies $A B=G$, let $G$ be the quotient of the Heisenberg group by a lattice subgroup of its center, let $A$ be a lattice in $G$, and let $\Gamma=e$. Then $B$ must be compact, so $B \subset Z(G)$.

Similarly, the conclusion can fail if $A \backslash G$ is not faithful. For example, let $G$ be the quotient of the Heisenberg group by a lattice subgroup of its center, let $A=Z(G)$, and let $\Gamma$ be a lattice in $G$. Then $G$ is the only syndetic hull of $\Gamma$, so $A \cap B=A \neq e$.

Proposition 3.6. [R, Prop. 2.5, p. 31 and Thm. 3.1, p. 29]. Let $\Gamma$ be a closed subgroup of a simply connected, nilpotent Lie group $G$. Then $\Gamma$ has a unique syndetic hull in $G$.

Corollary 3.7. Let $\Gamma$ be a closed subgroup of a connected, nilpotent Lie group $G$. Then $\Gamma$ has a syndetic hull in $G$, and has a unique maximal syndetic hull, which contains the maximal compact subgroup of $G$.

Proof. Because $G$ is nilpotent, it has a unique maximal compact subgroup $K$, and $K \triangleleft G$ (see 2.10). Then $G / K$ is simply connected (see 2.8 ), so the proposition implies that $\Gamma K / K$ has a unique syndetic hull $B / K$. The subgroup $B$ is a syndetic hull of $\Gamma$.

If $B^{\prime}$ is any syndetic hull of $\Gamma$, then $B^{\prime} K / K$ is a syndetic hull of $\Gamma K / K$, so the uniqueness of syndetic hulls in $G / K$ implies $B^{\prime} K / K=B / K$. Hence $B^{\prime} \subset B$, so $B$ is the unique maximal syndetic hull.

Theorem 3.8. Let $G$ be a nilpotent, transitive group of diffeomorphisms of $\mathbb{R}^{n}$. (Assume that $G$ is finite dimensional, and that $G$ is connected.) Then a strong form 
of the Bieberbach Condition (1.10) holds. Namely, if $\Gamma$ is a properly discontinuous subgroup of $G$, such that $\mathbb{R}^{n} / \Gamma$ is compact, then $\Gamma$ has a syndetic hull $B$, such that $B$ acts simply transitively on $\mathbb{R}^{n}$.

Proof. Let $B$ be a syndetic hull of $\Gamma$ in $G$ (see 3.7). The action of $G$ is faithful, so Theorem 3.4 asserts that $B$ is simply transitive on $\mathbb{R}^{n}$.

Theorem 3.9. Let $\Gamma$ be a properly discontinuous subgroup of a connected, nilpotent Lie group $G$ that acts transitively on $\mathbb{R}^{n}$. If $\mathbb{R}^{n} / \Gamma$ is compact, then there is some discrete subgroup $\Gamma^{\prime}$ of some Lie group $G^{\prime}$, such that some affine map from $G^{\prime} / \Gamma^{\prime}$ to $\mathbb{R}^{n} / \Gamma$ is a diffeomorphism.

Proof. There is no harm in assuming that the action of $G$ is faithful. Then, from the Bieberbach Condition (3.8), we know $\Gamma$ has a syndetic hull $B$ that acts simply transitively on $\mathbb{R}^{n}$. Then the affine map from $B / \Gamma$ to $\mathbb{R}^{n} / \Gamma$ that is induced by the inclusion of $B$ into $G$ is a diffeomorphism (see 2.3).

Definition 3.10 ([AGH], $[\mathrm{P}])$. As already mentioned in Definition 1.7, each oneparameter subgroup of $G$ defines a flow on any homogeneous space $G / \Gamma$ of $G$. If $G$ is nilpotent, these flows are called nilflows.

Corollary 3.11. Every geometric flow on a niltessellation $\mathbb{R}^{n} / \Gamma$ is isomorphic to a nilflow.

Proof. Let $A=G_{0}$ be the isotropy group of 0 in $G$, so $\mathbb{R}^{n}$ is $G$-equivariantly diffeomorphic to $A \backslash G$ (see 2.2). In accordance with Proposition 2.4, let $X$ be a Lie subgroup of $G$ that contains $A$ as a codimension-one, normal subgroup. There is no harm in assuming that the homogeneous space $A \backslash G$ is faithful, in which case, the Bieberbach Condition (3.8) asserts that $\Gamma$ is contained in a subgroup $B$ of $G$, such that $A B=G$ and $A \cap B=e$. Then Lemma 3.12 implies that the action of $X / A$ on $A \backslash G / \Gamma$ is isomorphic to the action of $X \cap B$ on $B / \Gamma$.

Lemma 3.12. Let $A$ and $\Gamma$ be closed subgroups of a Lie group $G$. Let $X$ be a Lie subgroup of $G$ that contains $A$ as a normal subgroup, and let $B$ be a closed subgroup of $G$ containing $\Gamma$, such that $A B=G$. Then the left action of $X / A$ on $A \backslash G / \Gamma$ is naturally isomorphic to the left action of $(X \cap B) /(A \cap B)$ on $(A \cap B) \backslash B / \Gamma$.

Proof. Since $\Gamma \subset B$ and $A B=G$, it is not difficult to see that the natural inclusion of $(A \cap B) \backslash B / \Gamma$ into $A \backslash G / \Gamma$ is a bijection. Clearly, it is $(X \cap B)$-equivariant, so the action of $(X \cap B) /(A \cap B)$ on $(A \cap B) \backslash B / \Gamma$ is isomorphic to the action of $(X \cap B) /(A \cap B)$ on $A \backslash G / \Gamma$. Since $A \subset X$ and $A B=G$, we have $(X \cap B) A=X$, so $(X \cap B) A / A=X / A$. Therefore, the action of $(X \cap B) /(A \cap B)$ on $A \backslash G / \Gamma$ is isomorphic to the action of $X / A$ on $A \backslash G / \Gamma$.

\section{Background from the Theory of Real Algebraic Groups}

Our proofs employ some basic properties of algebraic groups over $\mathbb{R}$, in the spirit of [R, $\S$ P.2, pp. 7-11]. For general background on algebraic groups, see a text such as $[\mathrm{B} 2]$ or $[\mathrm{Hu}]$.

Definition $4.1([\mathrm{~B} 2],[\mathrm{Hu}])$. A function $f$ on $\mathrm{GL}(n, \mathbb{R})$ is regular if there is a polynomial $p$ in $n^{2}+1$ variables, such that $f(g)=p\left(g_{i j}, \operatorname{det} g^{-1}\right)$. A subgroup $A$ of $\operatorname{GL}(n, \mathbb{R})$ is said to be Zariski closed (or we may say that $A$ is a real algebraic group) 
if $A$ is an algebraic subvariety of $\operatorname{GL}(n, \mathbb{R})$. That is, $A$ is the set of zeros of some collection of regular functions on $\operatorname{GL}(n, \mathbb{R})$.

As a subset of Euclidean space $\mathbb{R}^{n^{2}}$, a real algebraic group inherits a topology, in which it is a smooth manifold. When necessary to avoid confusion with the Zariski topology, we refer to this as the Euclidean topology.

Definition 4.2. A subgroup $A$ of $\mathrm{GL}(n, \mathbb{R})$ is said to be almost Zariski closed (or we may say that $A$ is an almost algebraic group) if there is a Zariski closed subgroup $B$ of $\mathrm{GL}(n, \mathbb{R})$, such that $B^{\circ} \subset A \subset B$, where $B^{\circ}$ is the identity component of $B$ in the Euclidean topology. The difference between being Zariski closed and almost Zariski closed is slight, because $B^{\circ}$ always has finite index in $B$ (see 4.15).

Definition 4.3 ([B2, $\S 4$ and $\S 8]$ ). A real algebraic group $U$ is unipotent if every element of $U$ is unipotent. A real algebraic group $T$ is a torus if $T$ is abelian and Zariski connected, and every element of $T$ is semisimple. (For convenience, we often also refer to the almost algebraic group $T^{\circ}$ as a torus.) A torus $T$ is $\mathbb{R}$-split if every element of $T$ is diagonalizable over $\mathbb{R}$.

Lemma 4.4 ([B2, Thm. 10.6, pp. 137-138]). Let $G$ be a Zariski-connected, solvable real algebraic group. Then:

1. the set $U$ of all unipotent elements of $G$ is a normal subgroup, which is called the unipotent radical of $G$;

2. for every maximal torus $T$ of $G$, we have $G=T \ltimes U$; and

3. $G / U$ is abelian.

Lemma 4.5 ([B2, Prop. 8.15 and Ex. 8.16]). Let $T$ be a Zariski closed torus. If $T_{\text {split }}$ is the maximal $\mathbb{R}$-split subtorus of $T$, and $T_{\text {cpct }}$ is the maximal compact subtorus of $T$, then $T=T_{\mathrm{split}} \cdot T_{\mathrm{cpct}}$, and $T_{\mathrm{split}} \cap T_{\mathrm{cpct}}$ is finite.

Example 4.6. The multiplicative group $\mathbb{R}^{\#}$ can be realized as a real algebraic group (in fact, an $\mathbb{R}$-split torus) via the embedding

$$
\mathbb{R}^{\#} \rightarrow \mathrm{GL}(2, \mathbb{R}): x \mapsto\left(\begin{array}{cc}
x & 0 \\
0 & 1 / x
\end{array}\right) .
$$

The subgroup $\mathbb{R}^{+}$of positive reals, being the identity component of $\mathbb{R}^{\#}$, is almost Zariski closed, but it is not Zariski closed.

In general, any Zariski-connected, solvable real algebraic group $B$ has a decomposition $B=\left(T_{\text {split }} T_{\text {cpct }}\right) \ltimes U$. With the identification

$$
T_{\text {split }}=\mathbb{R}^{\#} \times \mathbb{R}^{\#} \times \cdots \times \mathbb{R}^{\#},
$$

we have

$$
B=\left(\left(\mathbb{R}^{\#} \times \mathbb{R}^{\#} \times \cdots \times \mathbb{R}^{\#}\right) T_{\text {cpct }}\right) \ltimes U,
$$

and then

$$
B^{\circ}=\left(\left(\mathbb{R}^{+} \times \mathbb{R}^{+} \times \cdots \times \mathbb{R}^{+}\right) \times T_{\text {cpct }}\right) \ltimes U .
$$

Definition 4.7. The almost-Zariski closure $\bar{A}$ of a subgroup $A$ of an almost algebraic group is the unique smallest almost-Zariski closed subgroup that contains $A$.

In particular, if $A$ is a subgroup of a connected Lie group $G$, we use $\overline{\operatorname{Ad}_{G} A}$ to denote the almost-Zariski closure of $\operatorname{Ad}_{G} A$ in the real algebraic group $\operatorname{Aut}(\mathcal{G})$, where $\mathcal{G}$ is the Lie algebra of $G$. 
4A. The action of the adjoint group. If $G$ is simply connected, it is a basic fact of Lie theory that every group of automorphisms of the Lie algebra $\mathcal{G}$ lifts to a group of automorphisms of $G$ [V, Cor. 2.7.6, p. 72]. If $G$ is connected, but not simply connected, then, unfortunately, there may be automorphisms of $\mathcal{G}$ that do not lift to automorphisms of $G$. For example, $\operatorname{Aut}\left(\mathbb{T}^{n}\right)=\operatorname{GL}(n, \mathbb{Z})$ is not the full group $\mathrm{GL}(n, \mathbb{R})$ of automorphisms of the Lie algebra $\mathbb{R}^{n}$.

The following proposition shows that the group $\overline{\operatorname{Ad} G}$ does lift to a group of automorphisms of $G$, even if $G$ is not simply connected (see 4.8). Therefore, every Lie subgroup $S$ of $\overline{\operatorname{Ad} G}$ acts on $G$, so we may form the semidirect product $S \ltimes G$ of $S$ with $G$. In our most important applications of this sort, $G$ is solvable, and $S$ is a torus in $\overline{\operatorname{Ad} G}$.

Proposition 4.8 ([W2, §3]). If $G$ is a connected Lie group, then $\overline{\operatorname{Ad} G}$ acts on $G$, and centralizes $Z(G)$.

Lemma 4.9 ([W2, §3]). If $G$ is a connected Lie subgroup of $\mathrm{GL}(n, \mathbb{R})$, then, for every $\gamma \in \overline{\operatorname{Ad} G}$, there is some $g \in \bar{G}$, such that the action of $\gamma$ on $\mathcal{G}$ is the same as the action of $g$ on $\mathcal{G}$ by conjugation.

Lemma 4.10 ([W2, §3]). Let $A$ be a subgroup of a connected Lie group $G$. If nil $G$ is simply connected, then the centralizer of $A$ in $\overline{\operatorname{Ad} G}$ is Zariski closed.

Corollary 4.11 ([W2, §3]). Let $A$ be a connected Lie subgroup of a connected Lie group $G$. Then the centralizer of $A$ in $\overline{\operatorname{Ad} G}$ is Zariski closed.

Remark 4.12. The preceding two corollaries can fail if $A$ is not connected and nil $G$ is not simply connected. For example, let $\Gamma$ be a lattice in the Heisenberg group $H$, and let $G=H /[\Gamma, \Gamma]$ and $A=\Gamma /[\Gamma, \Gamma]$. Then $A$ centralizes $A$, but $\overline{\operatorname{Ad}_{G} A}=\overline{\operatorname{Ad} G}$ does not.

Lemma 4.13 (cf. proof of [Z, Thm. 3.2.5, p. 42]). Let $A$ be a connected Lie subgroup of an almost algebraic group $H$. Then $N_{H}(A)$ is Zariski closed.

Corollary 4.14. Let $A$ be a connected Lie subgroup of a connected Lie group $G$. Then the normalizer of $A$ in $\overline{\operatorname{Ad} G}$ is Zariski closed. In particular, $\overline{\operatorname{Ad}_{G} A}$ normalizes A.

4B. Miscellaneous facts. For ease of reference, we record a number of wellknown, useful results on real algebraic groups.

Lemma 4.15 ([M2, Appendix]). Any almost algebraic group has only finitely many connected components, in the Euclidean topology.

Lemma 4.16 (Chevalley [Ba, Thm. 16, p. 116]). Every compact subgroup of an almost algebraic group is Zariski closed.

Lemma 4.17 ([Hu, Cor. 7.4, p. 54]). If $A$ and $B$ are almost-Zariski closed subgroups of an almost algebraic group $H$, such that $A B$ is a subgroup, then $A B$ is almost Zariski closed.

Lemma 4.18 ([Bou, Cor. I.5.3.7, p. 47]). Let $A$ be a connected subgroup of a Lie group $G$. Then $A \subset \operatorname{nil} G$ if and only if $\overline{\operatorname{Ad}_{G} A}$ is unipotent. In particular, $\overline{\operatorname{Ad}_{G} \operatorname{nil} G}$ is unipotent.

Lemma 4.19. Every connected Lie subgroup of a unipotent, almost algebraic group is Zariski closed. 
Proof. Let $A$ be a connected Lie subgroup of a unipotent, almost algebraic group $U$, and let $\mathcal{U}$ be the Lie algebra of $U$. Because $U$ is unipotent, the exponential map $\exp : \mathcal{U} \rightarrow U$ is an isomorphism of varieties [Ho, Thm. VIII.1.1, p. 107], so, because the Lie algebra $\mathcal{A}$ of $A$ is a subspace of $\mathcal{U}$, hence Zariski closed, we know that $A=\exp \mathcal{A}$ is Zariski closed.

Proposition 4.20 ([Ho, pf. of Thm. XVIII.3.2, p. 220]). If $G$ is a connected, solvable Lie group, such that nil $G$ is simply connected, then $G$ has a faithful, finitedimensional representation $\rho$, such that $\rho(\operatorname{nil} G)$ is unipotent.

Lemma 4.21 ([B2, Thm. 19.2, p. 223]). In a connected, solvable, almost algebraic group, all the maximal tori are conjugate.

Corollary 4.22. Let $G$ be a connected, solvable Lie group. Then all the maximal tori of $\overline{\operatorname{Ad} G}$ are conjugate under $\operatorname{Ad} G$.

Proof. Let $T$ be a maximal torus of $\overline{\operatorname{Ad} G}$, and let $M=[T(\operatorname{Ad} G)] \cap U$, where $U$ is the unipotent radical of $\overline{\operatorname{Ad} G}$. Then $\operatorname{Ad} G \subset T M$, and $T M$ is Zariski closed (see 4.17), so we must have $T M=\overline{\operatorname{Ad} G}$. Thus, $M=U$, which means $U \subset T(\operatorname{Ad} G)$, so $T(\operatorname{Ad} G) \supset T U=\overline{\operatorname{Ad} G}$. From the lemma, we know that all maximal tori of $\overline{\operatorname{Ad} G}$ are conjugate to $T$ under $\overline{\operatorname{Ad} G}$, so this implies that all maximal tori of $\overline{\operatorname{Ad} G}$ are conjugate to $T$ under $\operatorname{Ad} G$.

Lemma 4.23. Let $A$ be a subgroup of a connected, solvable Lie group $G$. If $A$ nil $G=G$, then $\overline{\operatorname{Ad}_{G} A}$ contains a maximal torus of $\overline{\operatorname{Ad} G}$.

Proof. Let $S$ be a maximal torus of $\overline{\operatorname{Ad}_{G} A}$, and let $T$ be a maximal torus of $\overline{\operatorname{Ad} G}$ that contains $S$. Then $\overline{\operatorname{Ad} G}=T \ltimes U$, where $U$ is the unipotent radical of $\overline{\operatorname{Ad} G}$. Because $A($ nil $G)=G$ and $\overline{\operatorname{Ad}_{G} \text { nil } G} \subset U$ (see 4.18), we have $\overline{\operatorname{Ad}_{G} A} U=\overline{\operatorname{Ad} G}$, so $S U=\overline{\operatorname{Ad} G}$. Therefore, $S=T$.

4C. Syndetic hulls. Every closed subgroup of a connected, nilpotent Lie group has a syndetic hull (see 3.7), but this is not true for subgroups of solvable groups (see 4.24). Therefore, it is very useful to have a result that guarantees the existence of a syndetic hull in certain cases (see 4.25).

Example 4.24. Let us construct a tessellation whose fundamental group has no syndetic hull. Fix a real number $p$ with $0<p<1$, and define a 4-parameter group $G=\left\{\gamma_{a, b, c, d} \mid \in \mathbb{R}\right\}$ of diffeomorphisms of $\mathbb{R}^{2}$ by

$$
\gamma_{a, b, c, d}(x, y)=(x+a, y+b+p(c \cos 2 \pi x+d \sin 2 \pi x)) .
$$

Then $\left\{\gamma_{a, b, 0,0}\right\}$ is easily seen to be transitive on $\mathbb{R}^{2}$, so $G$ is transitive. The subgroup $\left\{\gamma_{0, b, c, d}\right\}$ is a codimension-one, abelian, normal subgroup, so $G$ is solvable. (More precisely, we have

$$
G \cong \mathbb{R} \ltimes\left(\mathbb{R} \times \mathbb{R}^{2}\right)
$$

where $\mathbb{R}$ centralizes $\mathbb{R} \times(0,0)$ and acts by rotation on $0 \times \mathbb{R}^{2}$.) Let

$$
\Gamma=\left\{\gamma_{m, n, n, 0} \in G \mid m, n \in \mathbb{Z}\right\} .
$$

For $m, n \in \mathbb{Z}$, we have

$$
\gamma_{m, n, n, 0}(x, y)=(x+m, y+n(1+p \cos 2 \pi x)),
$$

so it is clear that $\Gamma$ is a properly discontinuous subgroup of $G$, and that

$$
\{(x, y) \mid 0 \leq x \leq 1,0 \leq y \leq 1+p \cos 2 \pi x\}
$$




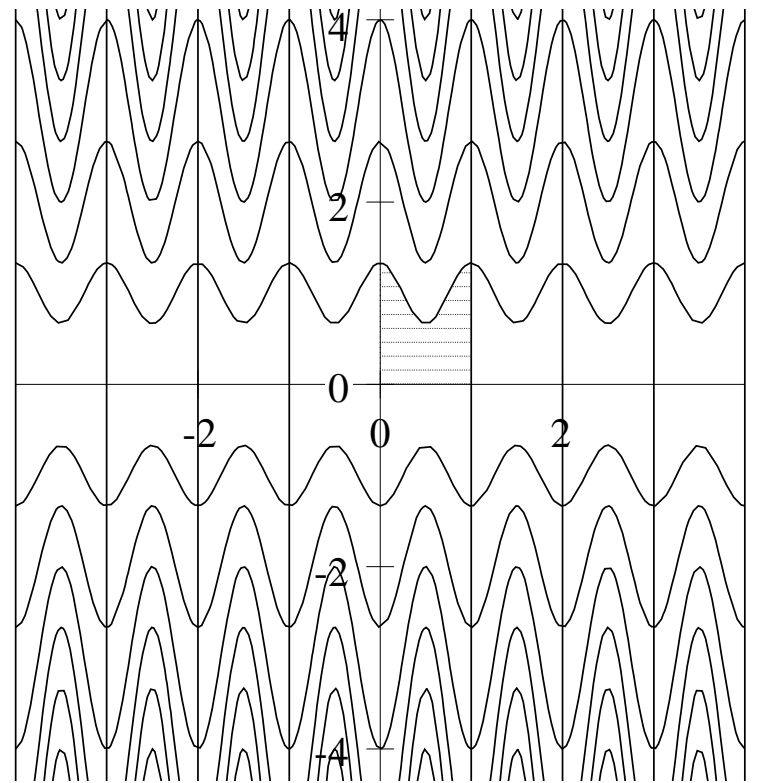

Figure 4.1. $\Gamma$-translates of a fundamental domain for the tessellation of Example 4.24 (with $p=1 / 3$ )

is a fundamental domain for the action of $\Gamma$ (see Figure 4.1), so $\mathbb{R}^{2} / \Gamma$ is compact.

However, we claim that no finite-index subgroup of $\Gamma$ has a syndetic hull in $G$. In fact, no finite-index subgroup of $\Gamma$ is contained in any connected, proper subgroup of $G$. (Thus, every finite-index subgroup of $\Gamma$ is "full" in $G$ [M1], [CW].) To see this, let $B$ be a connected subgroup of $G$ that contains a finite-index subgroup $\Gamma^{\prime}$ of $\Gamma$. Because nil $G=\left\{\gamma_{0, b, c, d}\right\}$, we have $\Gamma^{\prime} \not \subset \operatorname{nil} G$, so $(B \operatorname{nil} G) / \operatorname{nil} G$ is a nontrivial, connected subgroup of $G / \operatorname{nil} G \cong \mathbb{R}$. Hence $B \operatorname{nil} G=G$, so, since $\overline{\operatorname{Ad} G}$ is a torus, we have $\overline{\operatorname{Ad}_{G} B}=\overline{\operatorname{Ad} G}$ (see 4.23). Therefore, $B \triangleleft G$ (see 4.13), so

$$
B \supset[B, G] \supset\left[\Gamma^{\prime}, G\right]=\left\{\gamma_{0,0, c, d}\right\} .
$$

Now $\Gamma^{\prime}\left[\Gamma^{\prime}, G\right] /\left[\Gamma^{\prime}, G\right] \cong \mathbb{Z}^{2}$ is a lattice in $G /\left[\Gamma^{\prime}, G\right] \cong \mathbb{R}^{2}$, and so, because a syndetic hull in $\mathbb{R}^{2}$ is unique (see 3.6), we conclude that $B /\left[\Gamma^{\prime}, G\right]=G /\left[\Gamma^{\prime}, G\right]$. Hence, $B=G$, as claimed.

Proposition 4.25 ([W2, $\S 5])$. Let $\Gamma$ be a discrete subgroup of a connected, solvable Lie group $G$, and assume $\overline{\operatorname{Ad}_{G} \Gamma}$ contains a maximal compact torus of $\overline{\operatorname{Ad} G}$. Then $\Gamma$ has a syndetic hull in $G$. Furthermore, some finite-index subgroup of $\Gamma$ has a syndetic hull $B$ in $G$, such that $B /[B, B]$ is simply connected. Therefore, $[B, B]$ contains every compact subgroup of $B$.

Lemma 4.26. If $A$ is a closed subgroup of a solvable Lie group $G$, and $N$ is the maximal syndetic hull of $A \cap \operatorname{nil} G$ in nil $G$, then $\overline{\operatorname{Ad}_{G} A}$ normalizes $A N$.

Proof. Replacing $A$ with $A N$, we may assume $A \cap \operatorname{nil} G$ is connected, and contains the maximal compact subgroup $K$ of nil $G$. Then, by modding out $K$, we may assume nil $G$ is simply connected (see 2.8). Hence, $G$ has a faithful, finitedimensional representation that is unipotent on nil $G$ (see 4.20 ), so we may assume $G \subset \operatorname{GL}(n, \mathbb{R})$, and nil $G$ is unipotent. Then $A \cap \operatorname{nil} G$ is a connected subgroup 
of a unipotent group, so $\overline{A \cap \operatorname{nil} G}=A \cap \operatorname{nil} G$ (see 4.19). Therefore, because $[A, A] \subset A \cap \operatorname{nil} G$ (see 2.14), we have

$$
[A, \bar{A}] \subset \overline{[A, A]} \subset \overline{A \cap \operatorname{nil} G}=A \cap \operatorname{nil} G .
$$

Therefore, $[A, \bar{A}] \subset A$, so $\bar{A}$ normalizes $A$. Hence $\overline{\operatorname{Ad}_{G} A}$ normalizes $A$ (see 4.9).

4D. The Borel Density Theorem. The original theorem of Borel [B1] on Zariski density of lattice subgroups applied only to lattices in semisimple groups, but the result was generalized to all real algebraic groups by Dani [Da, Cor. 2.6]. Here, we need only the following special case of this for solvable groups.

Theorem 4.27 (Borel Density Theorem [W1, Cor. 4.2]). Let $\Gamma$ be a closed subgroup of a connected, solvable Lie group $G$, such that $G / \Gamma$ is compact. Then, for every finite-dimensional representation $\rho: G \rightarrow \mathrm{GL}(n, \mathbb{R})$, and every maximal compact torus $T_{\mathrm{cpct}}$ of $\overline{G^{\rho}}$, we have $\overline{G^{\rho}}=\overline{\Gamma^{\rho}} T_{\mathrm{cpct}}$.

Corollary 4.28. Let $\Gamma$ be a closed subgroup of a connected, solvable Lie group $G$, such that $\overline{\operatorname{Ad}_{G} \Gamma}$ contains a maximal compact torus of $\overline{\operatorname{Ad} G}$. Then $\overline{\operatorname{Ad}_{G} \Gamma}=\overline{\operatorname{Ad}_{G} B}$, for every syndetic hull $B$ of $\Gamma$.

The assumption that $G$ is solvable is not necessary in the following corollary, but we have no need for the more general result.

Corollary 4.29. Let $A$ and $B$ be subgroups of a connected, solvable Lie group $G$. If $A \backslash G / B$ is compact, then

$$
\overline{\operatorname{Ad}_{G} A} \operatorname{Ad} G \overline{\operatorname{Ad}_{G} B}=\overline{\operatorname{Ad} G} .
$$

Furthermore, for every compact set $K \subset \overline{\mathrm{Ad} G}$, there is a compact set

$$
K_{1} \subset \overline{\operatorname{Ad}_{G} A} \times G \times \overline{\operatorname{Ad}_{G} B}
$$

such that

$$
\forall s \in K, \exists(\alpha, g, \beta) \in K_{1} \text {, such that } s=\alpha(\operatorname{Ad} g) \beta .
$$

Proof. Because $A \backslash G / B$ is compact, we know $G /[A B($ nil $G)]$ is compact, so the Borel Density Theorem (4.27) implies that

$$
\frac{\overline{\operatorname{Ad} G}}{\overline{\operatorname{Ad}_{G} A} \overline{\operatorname{Ad}_{G} B} \overline{\operatorname{Ad}_{G} \operatorname{nil} G}}
$$

is a compact torus. Let $\tau$ be the natural homomorphism from $G$ into this torus. On the one hand, $G^{\tau}$ is Zariski dense, hence dense (see 4.16). On the other hand,

$$
A^{\tau}=B^{\tau}=(\operatorname{nil} G)^{\tau}=e,
$$

so the compactness of $G /[A B(\operatorname{nil} G)]$ implies $G^{\tau}$ is closed. Hence $\tau$ is surjective, so

$$
\overline{\operatorname{Ad}_{G} A} \overline{\operatorname{Ad}_{G} B} \overline{\operatorname{Ad}_{G} \operatorname{nil} G} \operatorname{Ad} G=\overline{\operatorname{Ad} G} .
$$

From Lemma 4.19, we know that $\overline{\operatorname{Ad}_{G} \operatorname{nil} G}=\operatorname{Ad}_{G} \operatorname{nil} G$, so $\overline{\operatorname{Ad}_{G} \operatorname{nil} G} \subset \operatorname{Ad} G$. Hence, we conclude that

$$
\overline{\operatorname{Ad}_{G} A} \overline{\operatorname{Ad}_{G} B} \operatorname{Ad} G=\overline{\operatorname{Ad} G} .
$$

Because $\operatorname{Ad} G$ is normal in $\overline{\operatorname{Ad} G}$ (see 4.13), we may rewrite this as

$$
\overline{\operatorname{Ad}_{G} A} \operatorname{Ad} G \overline{\operatorname{Ad}_{G} B}=\overline{\operatorname{Ad} G},
$$

as desired. 
We now know that the map

$$
\overline{\operatorname{Ad}_{G} A} \times \overline{\operatorname{Ad}_{G} B} \rightarrow \frac{\overline{\operatorname{Ad} G}}{\operatorname{Ad} G}
$$

is surjective. Because $\overline{\operatorname{Ad} G} /(\operatorname{Ad} G)$ is abelian (see 4.30), then it is easy to see that the map is a submersion. Hence the map

$$
\overline{\operatorname{Ad}_{G} A} \times G \times \overline{\operatorname{Ad}_{G} B} \rightarrow \overline{\operatorname{Ad} G}:(\alpha, g, \beta) \mapsto \alpha(\operatorname{Ad} g) \beta
$$

is a submersion. Therefore, the map has continuous local sections (see 4.31), so every compact subset of the range is the image of a compact subset of the domain, as desired.

Lemma 4.30. If $A$ is a connected, solvable Lie subgroup of an almost algebraic group $H$, then $\bar{A} / A$ is abelian.

Proof. Let $U$ be the unipotent radical of $\bar{A}$. Then $[\bar{A}, \bar{A}] \subset U$ (see 4.4), so $[A, A]$ is a subgroup of $U$. It is also connected, so $\overline{[A, A]}=[A, A]$ (see 4.19). Because $[\bar{A}, \bar{A}] \subset \overline{[A, A]}$, we conclude that $[\bar{A}, \bar{A}] \subset A$, as desired.

Lemma 4.31 (cf. [D, Thm. 10.3.1, p. 273]). If $X$ and $Y$ are $C^{\infty}$ manifolds, and $\phi: X \rightarrow Y$ is a $C^{\infty}$ submersion, then, for every $x \in X$, there is a neighborhood $U$ of $\phi(x)$, and a $C^{\infty}$ map $\psi: U \rightarrow X$, such that $\phi(\psi(y))=y$, for every $y \in U$. In other words, $\phi$ has $C^{\infty}$ local sections.

\section{The Nilshadow MaP}

Our proofs rely on the nilshadow construction, which was developed by Auslander and Tolimieri [AT]. In this section, we state the basic facts we need. For background and proofs, see [W2, §4].

Definition 5.1. Let $G$ be a connected, solvable Lie group, and let $T_{\text {cpct }}$ be a maximal compact torus of $\overline{\operatorname{Ad} G}$. There is a natural projection from $\overline{\operatorname{Ad} G}$ to $T_{\text {cpct }}$, given by the splitting $\overline{\operatorname{Ad} G}=\left(T_{\text {split }} \times T_{\text {cpct }}\right) \ltimes U$, where $T_{\text {split }}$ is any maximal $\mathbb{R}$ split torus and $U$ is the unipotent radical. Define $\pi: G \rightarrow T_{\text {cpct }}$ to be the composite homomorphism

$$
\pi: G \stackrel{\operatorname{Ad}}{\longrightarrow} \overline{\operatorname{Ad} G} \stackrel{\text { projection }}{\longrightarrow} T_{\text {cpct }} .
$$

Let $S$ and $S^{\perp}$ be almost complementary tori in $T_{\text {cpct }}$, by which we mean that $T_{\text {cpct }}=S S^{\perp}$, and $S \cap S^{\perp}$ is finite. Define $\tilde{T}_{\text {cpct }}=S \times S^{\perp}$, which is a finite cover of $T_{\text {cpct }}$. Assume that $\pi$ lifts to a homomorphism $\tilde{\pi}: G \rightarrow \tilde{T}_{\text {cpct. }}$. (For example, this is true if $G$ is simply connected.) Define $\sigma: G \rightarrow S$ to be the composite homomorphism

$$
\sigma: G \stackrel{\tilde{\pi}}{\longrightarrow} \tilde{T}_{\text {cpct }} \stackrel{\text { projection }}{\longrightarrow} S \stackrel{x \mapsto x^{-1}}{\longrightarrow} S .
$$

We define the nilshadow map $\Delta: G \rightarrow S \ltimes G$ by $\Delta(g)=\left(g^{\sigma}, g\right)$, where the action of $S$ on $G$ is given by Lemma 4.8 .

Warning: the nilshadow map is not a homomorphism (unless $S$ is trivial). In fact, a simple calculation shows that $\Delta$ is a crossed homomorphism (see 1.3), with respect to the homomorphism $-\sigma$. That is, $(a b)^{\Delta}=\left(a^{b^{-\sigma}}\right)^{\Delta} b^{\Delta}$, where $b^{-\sigma}=$ $\left(b^{-1}\right)^{\sigma}$. 
Assumption 5.2. For the remainder of this section, we assume $G$ is a connected, solvable Lie group, that choices of $T_{\text {cpct }}, S$, and $S^{\perp}$ have been made, and that the homomorphisms $\pi$ and $\sigma$ and the nilshadow map $\Delta$ have been defined as in Definition 5.1. (We assume that $\pi$ lifts to a homomorphism $\tilde{\pi}: G \rightarrow \tilde{T}_{\text {cpct }}$.)

Lemma 5.3. The nilshadow map $\Delta$ is a diffeomorphism onto its image. In particular, if $G$ is simply connected, then $G^{\Delta}$ is simply connected. If $A$ is a connected subset of $G$, then $A^{\Delta}$ is a connected subset of $G^{\Delta}$.

The following simple lemma is used to simplify calculations throughout the paper.

Lemma 5.4. For all $g \in G$ and $x \in \overline{\operatorname{Ad} G}$, we have $\left(g^{x}\right)^{\sigma}=g^{\sigma}$. In particular, $\left(a^{b^{\sigma}}\right)^{\sigma}=a^{\sigma}$, for all $a, b \in G$.

Corollary 5.5. Let $A$ be a subgroup of $G$. Then, for every $s \in S$, we have $\left(A^{\Delta}\right)^{s}=$ $\left(A^{s}\right)^{\Delta}$. In particular, $N_{S}(A)=N_{S}\left(A^{\Delta}\right)$.

Corollary 5.6. Let $A$ be a subgroup of $G$. Then $A^{\Delta}$ is a subgroup of $S \ltimes G$ iff $A^{\sigma}$ normalizes $A$.

Corollary 5.7. $G^{\Delta}$ is a normal subgroup of $S \ltimes G$.

Corollary 5.8. If $A$ is a connected subgroup of $G$ such that $A^{\sigma} \subset \overline{\operatorname{Ad}_{G} A}$, then $A^{\Delta}$ is a subgroup of $S \ltimes G$.

The following proposition shows that the nilshadow construction has killed precisely the torus $S$.

Proposition 5.9. Let $\phi: \overline{\operatorname{Ad} G} \rightarrow \operatorname{Aut}\left(\mathcal{G}^{\Delta}\right)$ be the homomorphism induced by the action of $G$ by conjugation on $G^{\Delta}$. Then ${\overline{\operatorname{Ad}} G^{\phi}}_{\text {contains }} \overline{\operatorname{Ad} G^{\Delta}}$ as a normal subgroup, and $\left(S^{\perp}\right)^{\phi}$ is a maximal compact torus of $\overline{\mathrm{Ad} G^{\Delta}}$.

The following technical result dictates our choice of $S$ in our proof of the Main Theorem (see $\S 7$ ).

Lemma 5.10. If $A$ is a subgroup of $G$ such that $\overline{\operatorname{Ad}_{G} A}$ is connected, and $T_{\text {cpct }}$ contains a maximal compact torus of $\overline{\operatorname{Ad}_{G} A}$, then $\overline{\operatorname{Ad}_{G} A}$ contains $A^{\pi}$, so $\overline{A^{\pi}}=$ $\overline{\operatorname{Ad}_{G} A} \cap T_{\text {cpct. }}$. If, in addition, $A$ is connected and

$$
\overline{A^{\pi}}=\left(\overline{A^{\pi}} \cap S\right)\left(\overline{A^{\pi}} \cap S^{\perp}\right),
$$

then $\overline{\operatorname{Ad}_{G} A}$ contains $A^{\sigma}$, so $A^{\Delta}$ is a subgroup of $G^{\Delta}$.

We now present a number of results showing that the structure of $G^{\Delta}$ mirrors some of the important structure of $G$.

Lemma 5.11. Let $A$ and $B$ be closed subgroups of $G$, such that $A^{\Delta} B^{\Delta}=G^{\Delta}$. If $S$ normalizes $A$, then $A B=G$.

Proof. Given any $g \in G$, there exist $a \in A$ and $b \in B$ such that

$$
\left(g^{\sigma}, g\right)=\left(a^{\sigma}, a\right) \cdot\left(b^{\sigma}, b\right)=\left((a b)^{\sigma}, a^{b^{\sigma}} b\right),
$$

so $g=a^{b^{\sigma}} b$. Because $b^{\sigma}$ normalizes $A$, this implies $g \in A B$, as desired.

Corollary 5.12. Let $A$ and $B$ be closed subgroups of $G$, such that $A^{\Delta} B^{\Delta}=G^{\Delta}$. If $S$ normalizes $B$, and $A^{\Delta}$ and $B^{\Delta}$ are subgroups, then $A B=G$. 
Proof. Because $A^{\Delta}$ and $B^{\Delta}$ are subgroups, they are closed under inverses; so, by taking inverses of the equation $A^{\Delta} B^{\Delta}=G^{\Delta}$, we obtain $B^{\Delta} A^{\Delta}=G^{\Delta}$. Then Lemma 5.11 (with the roles of $A$ and $B$ reversed) asserts that $B A=G$. Taking inverses again, we obtain $A B=G$.

Lemma 5.13. Let $A$ be a closed subgroup of $G$, such that $A^{\Delta}$ is a subgroup. If $A \backslash G$ is simply connected, then $\left(A^{\Delta}\right)^{s} \backslash G^{\Delta}$ is simply connected, for every $s \in S$.

Proof. Since $A \backslash G$ is simply connected, we know that $A$ is connected (see 2.11), so $\left(A^{\Delta}\right)^{s}$ is connected. Replacing $G$ by its universal cover, there is no harm in assuming $G$ is simply connected, which implies that $G^{\Delta}$ is simply connected. Hence $G^{\Delta}$ has no nontrivial compact subgroups (see 2.8 ), so $\left(A^{\Delta}\right)^{s}$ trivially contains the maximal compact subgroup of $G^{\Delta}$. Therefore, $\left(A^{\Delta}\right)^{s} \backslash G^{\Delta}$ is simply connected (see 2.11).

Proposition 5.14. Let $A$ and $B$ be closed subgroups of $G$, such that $A \backslash G / B$ is compact. Then $A^{\gamma} \backslash(S \ltimes G) / B$ is compact, for every $\gamma \in \overline{\operatorname{Ad} G}$.

Proof. Let $N_{A}$ and $N_{B}$ be the maximal syndetic hulls of $A \cap \operatorname{nil} G$ and $B \cap \operatorname{nil} G$, respectively, in nil $G$. Then $\overline{\operatorname{Ad}_{G} A}$ normalizes $A N_{A}$ and $\overline{\operatorname{Ad}_{G} B}$ normalizes $B N_{B}$ (see 4.26). Since $A$ and $B$ are cocompact in $A N_{A}$ and $B N_{B}$, there is no harm in replacing $A$ and $B$ with $A N_{A}$ and $B N_{B}$. Then $\overline{\operatorname{Ad}_{G} A}$ normalizes $A$, and $\overline{\operatorname{Ad}_{G} B}$ normalizes $B$.

From Lemma 4.29, we know that there is a compact set

$$
K_{1} \subset \overline{\operatorname{Ad}_{G} A} \times G \times \overline{\operatorname{Ad}_{G} B},
$$

such that

$$
\forall s \in S, \exists(\alpha, g, \beta) \in K_{1} \text {, such that } \gamma s=\alpha(\operatorname{Ad} g) \beta .
$$

By assumption, there is a compact set $K \subset G$ such that $A K B=G$. Let

$$
\mathcal{K}=\bigcup_{(\alpha, g, \beta) \in K_{1}}\left(g^{-1} K\right)^{\beta} .
$$

Then $\mathcal{K}$ is compact.

For every $s \in S$, there is some $(\alpha, g, \beta) \in K_{1}$, such that $\gamma s=\alpha g \beta$, so we have

$$
\begin{aligned}
A^{\gamma s} \mathcal{K} B & =A^{\alpha g \beta} \mathcal{K} B=A^{g \beta} \mathcal{K} B \supset A^{g \beta}\left(g^{-1} K\right)^{\beta} B=\left(A^{g}\left(g^{-1} K\right) B\right)^{\beta} \\
& =\left(g^{-1} A K B\right)^{\beta}=\left(g^{-1} G\right)^{\beta}=G^{\beta}=G .
\end{aligned}
$$

Therefore, for every $s \in S$, we have

$$
s G=s A^{\gamma s} \mathcal{K} B=A^{\gamma} s \mathcal{K} B \subset A^{\gamma} S \mathcal{K} B .
$$

Therefore $S \ltimes G=A^{\gamma} S \mathcal{K} B$. Since $S \mathcal{K}$ is compact, this implies $A^{\gamma} \backslash(S \ltimes G) / B$ is compact, as desired.

Corollary 5.15. Let $A$ and $B$ be closed subgroups of $G$, such that $A \backslash G / B$ is compact, and such that $A^{\Delta}$ and $B^{\Delta}$ are subgroups. Then $\left(A^{\Delta}\right)^{s} \backslash G^{\Delta} / B^{\Delta}$ is compact, for every $s \in S$.

Proof. From Proposition 5.14, we know that $A \backslash(S \ltimes G) / B$ is compact, which means there is a compact set $K$ such that $A K B=S \ltimes G$. Then, since $A \subset A^{\Delta} A^{\sigma}$ and $B \subset$ $B^{\sigma} B^{\Delta}$, we have $A^{\Delta} \overline{A^{\sigma}} K \overline{B^{\sigma}} B^{\Delta}=S \ltimes G$, so $A^{\Delta} \backslash(S \ltimes G) / B^{\Delta}$ is compact. Therefore, the closed subset $A^{\Delta} \backslash s G^{\Delta} / B^{\Delta}$ is compact, which implies $\left(A^{\Delta}\right)^{s} \backslash G^{\Delta} / B^{\Delta}$ is compact. 
Lemma 5.16. Let $A$ and $B$ be closed subgroups of $G$, such that $A \backslash G / B$ is compact, and such that $A^{\Delta}$ and $B^{\Delta}$ are subgroups. If the action of $B$ on $A \backslash G$ is proper, then the action of $B^{\Delta}$ on $\left(A^{\Delta}\right)^{s} \backslash G^{\Delta}$ is proper, for every $s \in S$.

Proof. This is similar to the proofs of Proposition 5.14 and Corollary 5.15.

Step 1. B is proper on $A \backslash(S \ltimes G)$. It suffices to show, for every compact set $K_{0} \subset S \ltimes G$, that $K_{0} A K_{0} \cap B$ is compact. Let $K \subset G$ be a compact set such that $K_{0} \subset S K \cap K S$. It suffices to show that $S K A K S \cap B$ is compact.

Let $N_{A}$ and $N_{B}$ be the maximal syndetic hulls of $A \cap \operatorname{nil} G$ and $B \cap \operatorname{nil} G$, respectively, in nil $G$. Then $\overline{\operatorname{Ad}_{G} A}$ normalizes $A N_{A}$ and $\overline{\operatorname{Ad}_{G} B}$ normalizes $B N_{B}$ (see 4.26). Since $A$ and $B$ are cocompact in $A N_{A}$ and $B N_{B}$, there is no harm in replacing $A$ and $B$ with $A N_{A}$ and $B N_{B}$. Then $\overline{\operatorname{Ad}_{G} A}$ normalizes $A$, and $\overline{\operatorname{Ad}_{G} B}$ normalizes $B$.

From Lemma 4.29 , we know that there is a compact set

$$
K_{1} \subset \overline{\operatorname{Ad}_{G} A} \times G \times \overline{\operatorname{Ad}_{G} B},
$$

such that

$$
\forall s \in S, \exists(\alpha, g, \beta) \in K_{1} \text {, such that } s=\alpha(\operatorname{Ad} g) \beta
$$

Let

$$
\mathcal{K}=\bigcup_{(\alpha, g, \beta) \in K_{1}}\left(g^{-1} K^{\alpha} \cup K^{\alpha} g\right) .
$$

For any $s \in S$, there is some $(\alpha, g, \beta) \in K_{1}$ with $s=\alpha(\operatorname{Ad} g) \beta$, so we have

$$
s^{-1} K A K s=\left(g^{-1} K^{\alpha} A^{\alpha} K^{\alpha} g\right)^{\beta} \subset(\mathcal{K} A \mathcal{K})^{\beta} .
$$

Since $B \subset G$, we have

$$
\begin{aligned}
S K A K S \cap B & =(S K A K S \cap G) \cap B=\left(\bigcup_{s \in S} s^{-1} K A K s\right) \cap B \\
& \subset\left(\bigcup_{(\alpha, g, \beta) \in K_{1}}(\mathcal{K} A \mathcal{K})^{\beta}\right) \cap B=\bigcup_{(\alpha, g, \beta) \in K_{1}}(\mathcal{K} A \mathcal{K} \cap B)^{\beta} .
\end{aligned}
$$

Since $B$ is proper on $A \backslash G, \mathcal{K} A \mathcal{K} \cap B$ is compact. Hence $\bigcup_{(\alpha, g, \beta) \in K_{1}}(\mathcal{K} A \mathcal{K} \cap B)^{\beta}$ is also compact.

Step 2. $B^{\Delta}$ is proper on $\left(A^{\Delta}\right)^{s} \backslash G^{\Delta}$. Since both $B$ and $B^{\Delta}$ are cocompact in $B \overline{B^{\sigma}}$, and $B$ is proper on $A \backslash(S \ltimes G)$, we know that $B^{\Delta}$ is proper on $A \backslash(S \ltimes G)$. Then, since both $A$ and $A^{\Delta}$ are cocompact in $A \overline{A^{\sigma}}$, we know that $B^{\Delta}$ is proper on $A^{\Delta} \backslash(S \ltimes G)$. Therefore, $B^{\Delta}$ is proper on the closed subset $A^{\Delta} \backslash s G^{\Delta}$, which implies that $B^{\Delta}$ is proper on $\left(A^{\Delta}\right)^{s} \backslash G^{\Delta}$.

Lemma 5.17. We have $\left[G^{\Delta}, G^{\Delta}\right] \subset[G, G]$.

Proof. Since $G^{\Delta} \subset T \ltimes G$, and $[T, T]=e$, it suffices to show $[T, G] \subset[G, G]$. Of course, $G$ centralizes $G /[G, G]$, which is the same as saying that $\operatorname{Ad} G$ centralizes $G /[G, G]$. Therefore $\overline{\operatorname{Ad} G}$ centralizes $G /[G, G]$ (cf. 4.10). Since $G \subset \overline{\operatorname{Ad} G}$, this implies $T$ centralizes $G /[G, G]$, which is the same as saying $[T, G] \subset[G, G]$, as desired. 


\section{The Syndetic Hull is Transitive}

Proposition 6.1. Let $\Gamma$ be a properly discontinuous subgroup of a connected, solvable Lie group $G$ that acts transitively on $\mathbb{R}^{n}$. If $\mathbb{R}^{n} / \Gamma$ is compact, then every syndetic hull of $\Gamma$ is transitive on $\mathbb{R}^{n}$.

If $B$ is a syndetic hull of a properly discontinuous subgroup $\Gamma$, then $B$ acts properly, so all $B$-orbits are closed. Therefore, the preceding proposition is a consequence of the following theorem, which is the main result of this section.

Theorem 6.2. Let $B$ be a connected Lie subgroup of a connected, solvable Lie group $G$ that acts transitively on $\mathbb{R}^{n}$. If $\mathbb{R}^{n} / B$ is compact, and every $B$-orbit is closed, then $B$ is transitive on $\mathbb{R}^{n}$.

The following example shows that the assumption that $B$-orbits are closed cannot be eliminated from the theorem.

Example 6.3. Let $G=\mathbb{R}^{+} \ltimes \mathbb{R}^{n}$, where $\mathbb{R}^{+}$acts by scalar multiplication, and $\mathbb{R}^{n}$ acts by translations. Then $\mathbb{R}^{n} / \mathbb{R}^{+} \approx S^{n-1} \cup\{0\}$ is compact, but $B$ is not transitive. In fact, $B$ is very far from being transitive; there are uncountably many $B$-orbits.

Note that, in the preceding example, 0 is in the closure of every $B$-orbit. The following conjecture asserts that all compact quotients share this behavior.

Conjecture 6.4. Let $B$ be a connected Lie subgroup of a connected, solvable Lie group $G$ that acts transitively on $\mathbb{R}^{n}$. If $\mathbb{R}^{n} / B$ is compact, then there is a unique closed $B$-orbit on $\mathbb{R}^{n}$, and the closure of every $B$-orbit contains this closed orbit.

For purposes of the proof, it is more convenient to state the theorem in the following group-theoretic terms.

Theorem 6.2'. Let $A \backslash G$ be a simply connected homogeneous space of a connected, solvable Lie group $G$. If $B$ is a connected subgroup of $G$, such that $A \backslash G / B$ is compact, and every $B$-orbit on $A \backslash G$ is closed, then $B$ is transitive on $A \backslash G$.

Theorem 6.2". Let $A$ and $B$ be connected subgroups of a connected, simply connected, solvable Lie group $G$. If $A \backslash G / B$ is compact, and every double coset $A g B$ is closed, then $A B=G$.

Before beginning the proof, we state the following simple fact.

Lemma 6.5. Let $A$ and $H$ be subgroups of a semidirect product $T \ltimes U$. If $T$ is contained in both $A$ and $H$ then, letting $U_{H}=H \cap U$, we have $H=T \ltimes U_{H}$ and $A \cap H=T \ltimes\left(A \cap U_{H}\right)$.

Proof of Theorem $\mathbf{6 . 2}^{\prime \prime}$. Let $A$ and $B$ be connected subgroups of a connected, simply connected, solvable Lie group $G$, such that $A \backslash G / B$ is compact. To detour around a technical difficulty that arises in Case 2 of the proof, we will not always assume that every point in $A \backslash G / B$ is closed. Instead, we assume that either

1. $\overline{\operatorname{Ad} G}$ is $\mathbb{R}$-split, and the closure of each point of $A \backslash G / B$ is a minimal closed subset; or

2. every point of $A \backslash G / B$ is closed.

Under this weaker hypothesis, we show that $G=A B$.

For convenience, let $U=\operatorname{nil} G$. Because $G / U$ is abelian (see 2.14), we know $G /(A U B)$ is an abelian group. It is compact, because $A \backslash G / B$ is compact, and it 
is simply connected, because $G$ is simply connected and $A U B$ is connected (see 2.11 and 2.8). Therefore, $G / A U B$ is trivial (see 2.7), that is, $G=A U B$. Hence, by Lemma 2.12, we may assume $U B=G$. Similarly, we may also assume $A U=G$.

Let $\bar{T}_{A}$ and $\bar{T}_{B}$ be maximal tori in $\overline{\operatorname{Ad}_{G} A}$ and $\overline{\operatorname{Ad}_{G} B}$, respectively. Since $A U=$ $B U=G$, we know that $\bar{T}_{A}$ and $\bar{T}_{B}$ are maximal tori in $\overline{\operatorname{Ad} G}$ (see 4.23). Then, replacing $B$ by a conjugate, we may assume $\bar{T}_{A}=\bar{T}_{B}$ (see 4.22 ).

Case 1. $\overline{\operatorname{Ad} G}$ is $\mathbb{R}$-split, and the closure of each point of $A \backslash G / B$ is a minimal closed subset.

Step 1.1. Let $U_{A}=U \cap A$ and $U_{B}=U \cap B$. We may assume there is a subgroup $T$ of $G$ such that $\overline{\operatorname{Ad}_{G} T}$ is a torus, and such that $G=T \ltimes U$ and $A=T \ltimes U_{A}$. Furthermore, we may assume $B=T_{B} \ltimes U_{B}$, where $T_{B}=\left\{t \cdot t^{\alpha} \mid t \in T\right\}$, for some homomorphism $\alpha: T \rightarrow Z(G)$. Since $\bar{T}_{A}$ and $\bar{T}_{B}$ are equal, it is obvious that they centralize each other, so we may form the semidirect product $\left(\bar{T}_{A} \times \bar{T}_{B}\right) \ltimes G$, and we may embed $\bar{T}_{A} \ltimes A$ and $\bar{T}_{B} \ltimes B$ as subgroups of $\left(\bar{T}_{A} \times \bar{T}_{B}\right) \ltimes G$ via the natural embeddings $(t, a) \mapsto(t, e, a)$ and $(t, b) \mapsto(e, t, b)$, respectively. It is straightforward to verify that the natural inclusion

$$
A \backslash G / B \rightarrow\left(\bar{T}_{A} \ltimes A\right) \backslash\left(\left(\bar{T}_{A} \times \bar{T}_{B}\right) \ltimes G\right) /\left(\bar{T}_{B} \ltimes B\right)
$$

is a homeomorphism. Thus, there is no harm in replacing $G, A$, and $B$ by $\left(\bar{T}_{A} \times \bar{T}_{B}\right) \ltimes$ $G, \bar{T}_{A} \ltimes A$, and $\bar{T}_{B} \ltimes B$, respectively. Then, letting $T=\bar{T}_{A}$ and $\alpha(t)=\left(t^{-1}, t, e\right)$, we have the desired conclusions.

Step 1.2. We may assume $U_{A} T^{\alpha} U_{B}$ is a codimension-one subgroup of $U$. First, suppose $Z(G) U_{B}=U$. Then $U_{B}$ is normalized by $U$. Since $B=T_{B} \ltimes U_{B}$, we also know that $U_{B}$ is normalized by $T_{B}$. Hence $U_{B} \triangleleft G$, so we can $\bmod$ it out. Then $U=Z(G)$, from which it follows that $G$ is nilpotent (in fact, $G$ is abelian), so Corollary 3.2 implies $A B=G$, which completes the proof of the theorem.

We may now assume $Z(G) U_{B}$ is a proper subgroup of $U$. Because $U$ is nilpotent, this implies that $Z(G) U_{B}[U, U]$ is a proper subgroup of $U$ [Ha, Cor. 10.3.3, p. 155], so

$$
Z(G) U_{B}[U, U] /[U, U]
$$

is a proper subgroup of the abelian group $U /[U, U]$. It is $T$-invariant, so, because $\overline{\operatorname{Ad}_{G} T}$ is $\mathbb{R}$-split, we see that $Z(G) U_{B}[U, U] /[U, U]$ is contained in a $T$-invariant, codimension-one, connected subgroup $M /[U, U]$. We may assume that $M$ contains $U_{A}$, for otherwise the codimension of $M$ implies $U_{A} M=U$, from which it follows that $A(T M)=G$, so, by Lemmas 2.12 and 6.5 , we could replace $G$ with the proper subgroup $T M$. Now $T M$ is closed, so $A \backslash(T M) / B$ is a closed subset of $A \backslash G / B$, so $A \backslash T M / B$ is compact. Therefore, by induction on $\operatorname{dim} G$, we know that $A B=T M$. By intersecting with $M$, we conclude that $U_{A} T^{\alpha} U_{B}=M$.

Step 1.3. Let $M=U_{A} T^{\alpha} U_{B}$. There is a T-invariant, one-parameter subgroup $Y$ complementary to $M$ in $U$, and $Y$ is not centralized by $T$. The existence of $Y$ is a consequence of the fact that $\overline{\operatorname{Ad}_{G} T}$ is diagonalizable, and the fact that $M$ is a codimension-one, connected, $T$-invariant subgroup of $U$. Suppose $T$ centralizes $Y$. Then $T M$ is normalized by $T, M$, and $Y$, so $T M$ is normal in $G$. Since $A, B \subset T M$, and $A \backslash G / B$ is compact, this implies $G /(T M)$ is compact. However, we know $G /(T M) \approx Y \approx \mathbb{R}$. This is a contradiction. 
Step 1.4. We have $U_{A} y T^{\alpha} U_{B}=M y$, for every $y \in Y$. Let

$$
Y_{0}=\left\{y \in Y \mid U_{A} y T^{\alpha} U_{B}=M y\right\} .
$$

We wish to show $Y_{0}=Y$. Because $M \triangleleft U$, we have $M y=y M$, so

$$
Y_{0}=\left\{y \in Y \mid U_{A}^{y} T^{\alpha} U_{B}=M\right\} .
$$

For every $t \in T$ and $y \in Y$, we have

$$
\left(U_{A}^{y} T^{\alpha} U_{B}\right)^{t}=U_{A}^{y^{t}} T^{\alpha} U_{B} \text { and } M^{t}=M
$$

so we conclude that $Y_{0}$ is $T$-invariant. From the definition of $M$, we also know that $e \in Y_{0}$. We will show that $Y_{0}$ is open. No proper, open subset of the real line contains 0 and is invariant under multiplication by $\mathbb{R}^{+}$, so this implies $Y_{0}=Y$, as desired.

Since $U$ is a simply connected, nilpotent Lie group, it can be given the structure of a unipotent real algebraic group [V, Thm. 3.6.6, p. 199], and every connected subgroup of $U$ is Zariski closed (see 4.19). Thus, the homogeneous space $\mathcal{U}=$ $\left(U_{A} T^{\alpha}\right) \backslash U$ is an affine variety [B2, Cor. 6.9b, p. 99]. Define the map $\mu: Y U_{B} \rightarrow \mathcal{U}$ by $(y u)^{\mu}=U_{A} T^{\alpha} y u$. Then

$$
Y_{0}=\left\{y \in Y \mid y^{\mu} U_{B}=\left(U_{A} T^{\alpha}\right) \backslash M y\right\} .
$$

Since $M \triangleleft U$, and $U_{B} \subset M$, we have $\left(y U_{B}\right)^{\mu} \subset\left(U_{A} T^{\alpha}\right) \backslash M y$, for every $y \in Y$, so, because every $U_{B}$-orbit on $\mathcal{U}$ is Zariski closed [B2, Prop. 4.10, p. 88], we have

$$
\begin{aligned}
Y_{0} & =\left\{y \in Y \mid \operatorname{dim}\left(y^{\mu} U_{B}\right) \geq \operatorname{dim}\left(\left(U_{A} T^{\alpha}\right) \backslash M y\right)\right\} \\
& =\left\{y \in Y \mid \operatorname{dim} U_{B}-\operatorname{dim}\left(\left(U_{A} T^{\alpha}\right)^{y} \cap U_{B}\right) \geq \operatorname{dim}\left(\left(U_{A} T^{\alpha}\right) \backslash M\right)\right\} \\
& =\left\{y \in Y \mid \operatorname{dim} U_{B}-\operatorname{dim}\left(\mu^{-1}\left(y^{\mu}\right)\right) \geq \operatorname{dim}\left(\left(U_{A} T^{\alpha}\right) \backslash M\right)\right\} .
\end{aligned}
$$

Since $\operatorname{dim}\left(\mu^{-1}\left(y^{\mu}\right)\right)$ is an upper semicontinuous function of $y[\mathrm{Hu}$, Prop. I.4.4, p. 33], we conclude that $Y_{0}$ is an open subset of $Y$, as desired.

Step 1.5. The double-coset space $A \backslash G / B$ is homeomorphic to the space of equivalence classes of the equivalence relation on $Y T^{\alpha}$ defined by

$$
y^{\prime} z^{\prime} \sim y z \Leftrightarrow \exists a \in A, \exists b \in B \text {, such that } y^{\prime} z^{\prime}=a y z b .
$$

From Step 1.4, we know that $U_{A} y T^{\alpha} U_{B}=M y$, for every $y \in Y$; so $U_{A} Y T^{\alpha} U_{B}=U$, and hence $A Y T^{\alpha} B=G$. Therefore, the map $Y T^{\alpha} \rightarrow A \backslash G / B: x \mapsto A x B$ is surjective. The conclusion follows.

The rest of the proof of Case 1 is a study of these equivalence classes.

Step 1.6. We have

(6.1.6a) $y^{\prime} z^{\prime} \sim y z \Leftrightarrow \exists t \in T, \exists w \in U_{A}^{y^{\prime}} U_{B} \cap T^{\alpha}$, such that $y^{\prime}=y^{t}$ and $z^{\prime}=z t^{\alpha} w$.

$(\Rightarrow)$ Since $A=T \ltimes U_{A}$ and $B=T_{B} \ltimes U_{B}$, there exist $a \in T, u \in U_{A}, b \in T_{B}$, and $v \in U_{B}$, such that $y^{\prime} z^{\prime}=(a u) y z(b v)$. We may write $b=t t^{\alpha}$ for some $t \in T$. By considering the equation $y^{\prime} z^{\prime}=(a u) y z(b v)$ modulo $U$, we see that we must have $a=t^{-1}$, so

$$
y^{\prime} z^{\prime}=(a u) y z(b v)=y^{t} u^{t y^{t}} z t^{\alpha} v .
$$

Considering this equation modulo $M$, we see that we must have $y^{\prime}=y^{t}$, so $z^{\prime}=$ $u^{t y^{\prime}} z t^{\alpha} v$. Therefore, $u^{t y^{\prime}} v \in T^{\alpha}$. Since $T$ normalizes $U_{A}$, we also have $u^{t y^{\prime}} v \in$ $U_{A}^{y^{\prime}} U_{B}$. Thus, letting $w=u^{t y^{\prime}} v$, we obtain the desired conclusion. 
$(\Leftarrow)$ Write $w=u^{y^{\prime}} v$, with $u \in U_{A}$ and $v \in U_{B}$. Then, reversing the steps of the preceding paragraph, we have $y^{\prime} z^{\prime}=\left(t^{-1} u\right) y z\left(t T^{\alpha} v\right)$, so $y^{\prime} z^{\prime} \sim y z$.

Step 1.7. Let $Z=T^{\alpha} / C_{T}(Y)^{\alpha}$. Each equivalence class of $\sim$ is a union of equivalence classes of the equivalence relation $\sim_{T}$ on $Y Z$ defined by

$$
y^{\prime} z^{\prime} \sim_{T} y z \Leftrightarrow \exists t \in T \text {, such that } y^{\prime}=y^{t} \text { and } z^{\prime}=z t^{\alpha} .
$$

Setting $w=e$ in the RHS of (6.1.6a), we have

$$
y^{\prime} z^{\prime} \sim y z \Leftarrow \exists t \in T \text {, such that } y^{\prime}=y^{t} \text { and } z^{\prime}=z t^{\alpha} .
$$

In particular, for any $t \in C_{T}(Y)$, we see that $y z t^{\alpha} \sim y z$. Therefore, each $\sim$ equivalence class is a union of cosets of $C_{T}(Y)^{\alpha}$. That is, $\sim$ induces an equivalence relation on $Y Z$, with the same equivalence classes. From (6.1.7b), we see that $g \sim_{T} h \Rightarrow g \sim h$, as desired.

Step 1.8. The subgroup $Z$ is not one-dimensional. Suppose, for a contradiction, that $Z$ is one-dimensional. Then $Y Z \approx Y \times Z$ can be identified with $\mathbb{R}^{2}$, and the $\sim_{T}$-equivalence classes are logarithmic curves (and the $Z$-axis), as illustrated in Figure 6.1. It is not difficult to see that the space of $\sim_{T}$-equivalence classes is not compact. (For example, each equivalence class $E$ in the right half-plane is the boundary of two open subsets of the plane; let $O_{E}$ be the one these subsets that contains the entire left half-plane. Then each $O_{E}$ is a union of equivalence classes, and $\left\{O_{E}\right\}$ is an increasing chain of open sets whose union is the whole plane.) Therefore, the $\sim$-equivalence classes cannot be the same as the $\sim_{T}$-equivalence classes; some of the $\sim_{T}$-equivalence classes must be combined into a single $\sim$ equivalence class. By comparing (6.1.6a) and (6.1.7a), and noting that $\{y=e\}$ is a single $\sim_{T}$-equivalence class, so cannot be collapsed any further, we see that there is some $y \neq e$ such that $U_{A}^{y} U_{B} \cap Z \neq e$. Because this intersection is a Zariski closed subgroup, and $Z$ is one-dimensional, then this intersection must be all of $Z$. Thus, for every $z \in Z$, we have $y \sim y z$. In terms of Figure 6.1 , this means that all of the points on the vertical line through $y$ are in the same $\sim$-equivalence class, which means that all of the $\sim_{T}$-equivalence classes that meet this vertical line are combined into a single $\sim$-equivalence class. It is clear from Figure 6.1 that this implies the (open) half-plane containing $y$ and bounded by the $Z$-axis is a single equivalence class. The closure of this half-plane clearly contains the $Z$-axis, which is a closed equivalence class. Thus, there is a non-closed point in $A \backslash G / B$ whose closure contains a closed point. This contradicts our assumption that the closure of every point is a minimal closed set.

Step 1.9. The final contradiction. Since $C_{T}(Y)$ has codimension 1 in $T$, the codimension of $C_{T}(Y)^{\alpha}$ in $T^{\alpha}$ must be $\leq 1$, so $\operatorname{dim} Z \leq 1$. From Step 1.8, we know $\operatorname{dim} Z \neq 1$, so we conclude that $Z$ is trivial. Then $Y Z=Y \approx \mathbb{R}$, and there are precisely three $\sim_{T}$-equivalence classes, namely $\mathbb{R}^{+}, 0$, and $\mathbb{R}^{-}$. The closure of $\mathbb{R}^{+}$ contains the closed point 0 , so this contradicts our assumption that the closure of every point is a minimal closed set. This completes the proof of Case 1.

Case 2. The general case. Let $T_{\text {cpct }}$ be a maximal compact torus of $\bar{T}_{A}$, define $S=T_{\text {cpct }}$ and $S^{\perp}=e$, and let $\Delta: G \rightarrow T_{\text {cpct }} \ltimes G$ be the corresponding nilshadow map, as described in Definition 5.1.

Since $T_{\text {cpct }} \subset \bar{T}_{A}=\bar{T}_{B}$, we know that $T_{\text {cpct }}$ normalizes both $A$ and $B$ (see 4.13). Therefore, $A^{\Delta}$ and $B^{\Delta}$ are subgroups of $G^{\Delta}$ (see 5.6), and $A^{\Delta} \backslash G^{\Delta} / B^{\Delta}$ is compact (see 5.15). Because $S^{\perp}=e$, we know that $\overline{\operatorname{Ad} G^{\Delta}}$ has no compact tori (see 5.9), so 


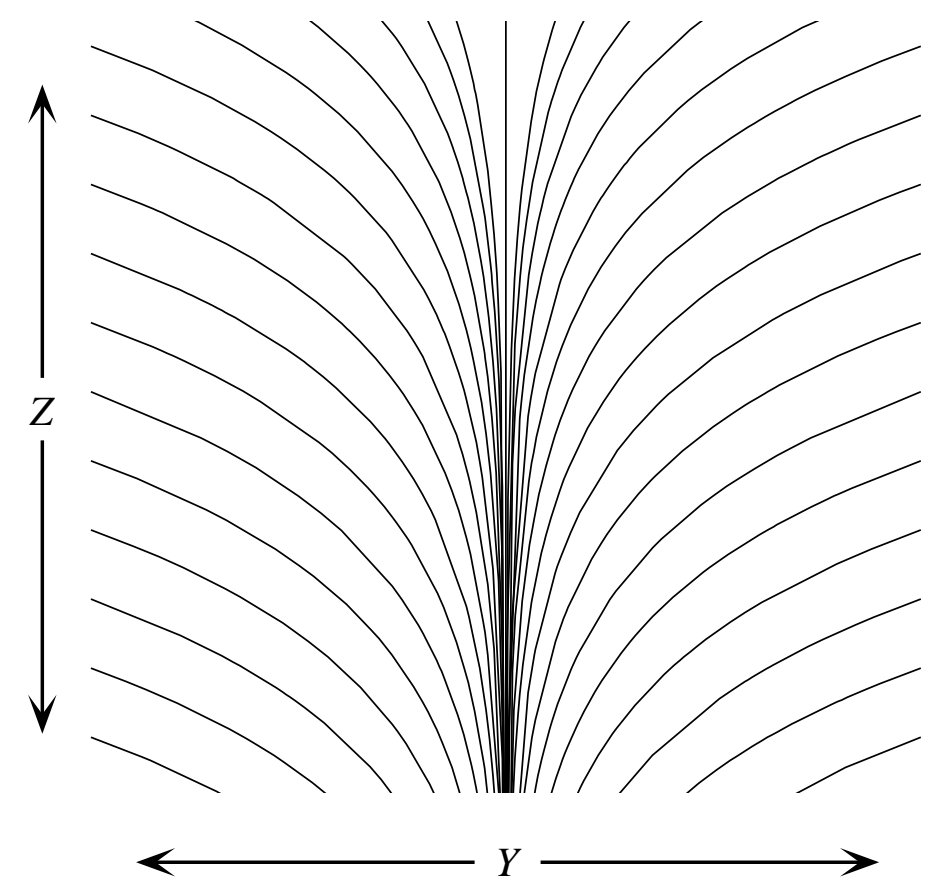

Figure 6.1. Equivalence classes of $\sim_{T}$

$\overline{\operatorname{Ad} G^{\Delta}}$ is $\mathbb{R}$-split. We now would like to invoke Case 1 to conclude that $A^{\Delta} B^{\Delta}=$ $G^{\Delta}$, for then Lemma 5.11 would imply $A B=G$, as desired. Thus, we now show that the closure of each point in $A^{\Delta} \backslash G^{\Delta} / B^{\Delta}$ is a minimal closed subset, which is sufficient to apply Case 1 , and conclude that $A^{\Delta} B^{\Delta}=G^{\Delta}$, as desired. (It does not seem to be obvious that all the double cosets $A^{\Delta}\left(g^{\sigma}, g\right) B^{\Delta}$ are closed. This is the technical difficulty mentioned at the beginning of the proof.)

Given $g \in G$, we wish to show that no nonempty, proper, closed subset of the closure of $A^{\Delta}\left(g^{\sigma}, g\right) B^{\Delta}$ is a union of double cosets. It suffices to show, for every $\left(h^{\sigma}, h\right)$ in the closure of $A^{\Delta}\left(g^{\sigma}, g\right) B^{\Delta}$, that the closure of $A^{\Delta}\left(h^{\sigma}, h\right) B^{\Delta}$ contains $\left(g^{\sigma}, g\right)$.

Step 2.1. There is some $t \in T_{\text {cpct }}$ such that $A^{\Delta}\left(h^{\sigma}, h\right) B^{\Delta}=A^{\Delta}\left(g^{\sigma}, g\right)^{t} B^{\Delta}$. There are sequences $\left\{a_{n}\right\} \subset A$ and $\left\{b_{n}\right\} \in B$ such that $\left(a_{n}^{\sigma}, a_{n}\right)\left(g^{\sigma}, g\right)\left(b_{n}^{\sigma}, b_{n}\right) \rightarrow$ $\left(h^{\sigma}, h\right)$, as $n \rightarrow \infty$. Since $T_{\text {cpct }}$ is compact, we may assume $a_{n}^{\sigma}$ converges to some $a^{\infty}$, and $b_{n}^{\sigma}$ converges to some $b^{\infty}$. We have

$$
\begin{aligned}
\left(h^{\sigma}, h\right) & \doteq\left(a_{n}^{\sigma}, a_{n}\right)\left(g^{\sigma}, g\right)\left(b_{n}^{\sigma}, b_{n}\right) \\
& =\left(a_{n}^{\sigma} g^{\sigma} b_{n}^{\sigma}, a_{n}^{g^{\sigma} b_{n}^{\sigma}} g^{b_{n}^{\sigma}} b_{n}\right) \\
& =\left(a_{n}^{\sigma} g^{\sigma} b_{n}^{\sigma},\left(a_{n}^{g^{\sigma}} g b_{n}^{\left(b_{n}^{\sigma}\right)^{-1}}\right)^{b_{n}^{\sigma}}\right) \\
& \doteq\left(a^{\infty} g^{\sigma} b^{\infty},\left(a_{n}^{g^{\sigma}} g b_{n}^{\left(b_{n}^{\sigma}\right)^{-1}}\right)^{b^{\infty}}\right) .
\end{aligned}
$$

Since $A g B$ is closed, and $T_{\text {cpct }}$ normalizes $A$ and $B$, this means that $h$ must be of the form $h=\left(a^{\prime} g b^{\prime}\right)^{b^{\infty}}$, for some $a^{\prime} \in A$ and $b^{\prime} \in B$. To simplify notation in the following calculations, choose $a \in A$ with $a^{g^{\sigma}}=a^{\prime}$ and let $b=\left(b^{\prime}\right)^{b^{\infty}}$. Then 
$h=a^{g^{\sigma} b^{\infty}} g^{b^{\infty}} b$. We have

$$
\begin{aligned}
a^{\infty} g^{\sigma} b^{\infty}= & h^{\sigma}=\left(a^{g^{\sigma} b^{\infty}} g^{b^{\infty}} b\right)^{\sigma}=a^{\sigma} g^{\sigma} b^{\sigma}, \\
\text { so }\left(a^{\infty}\left(a^{\sigma}\right)^{-1}\right)\left(b^{\infty}\left(b^{\sigma}\right)^{-1}\right)=e . & \text { Let } t=b^{\infty}\left(b^{\sigma}\right)^{-1}, \text { so } t^{-1}=a^{\infty}\left(a^{\sigma}\right)^{-1} \text {. Then } \\
\left(h^{\sigma}, h\right) & =\left(a^{\infty} g^{\sigma} b^{\infty}, a^{g^{\sigma} b^{\infty}} g^{b^{\infty}} b\right) \\
& =\left(a^{\infty}, a\right)\left(g^{\sigma}, g\right)\left(b^{\infty}, b\right) \\
& =\left(t^{-1} a^{\sigma}, a\right)\left(g^{\sigma}, g\right)\left(t b^{\sigma}, b\right) \\
& =\left(a^{\sigma}, a\right)^{t}\left(g^{\sigma}, g\right)^{t}\left(b^{\sigma}, b\right) \\
& \in A^{\Delta}\left(g^{\sigma}, g\right)^{t} B^{\Delta} .
\end{aligned}
$$

Step 2.2. The double coset of $\left(h^{\sigma}, h\right)$ contains $\left(g^{\sigma}, g\right)$ in its closure. We know from Step 2.1 (and the choice of $\left(h^{\sigma}, h\right)$ ) that the double coset of $\left(g^{\sigma}, g\right)$ contains $\left(g^{\sigma}, g\right)^{t}$ in its closure. Applying conjugation by $t^{m}$, which is a homeomorphism of $A^{\Delta} \backslash G^{\Delta} / B^{\Delta}$, to this statement, we conclude that the double coset of $\left(g^{\sigma}, g\right)^{t^{m}}$ contains $\left(g^{\sigma}, g\right)^{t^{m+1}}$ in its closure. By transitivity, then the double coset of $\left(g^{\sigma}, g\right)^{t^{m}}$ contains $\left(g^{\sigma}, g\right)^{t^{n}}$ in its closure, whenever $m<n$. Let $m=1$. Since $T_{\text {cpct }}$ is compact, we can choose a sequence $\left\{n_{i}\right\} \rightarrow \infty$ such that $t^{n_{i}} \rightarrow e$. Passing to the limit, we conclude that the double coset of $\left(g^{\sigma}, g\right)^{t}$ contains $\left(g^{\sigma}, g\right)$ in its closure. From Step 2.1, we know the double coset of $\left(h^{\sigma}, h\right)$ is the same as the double coset of $\left(g^{\sigma}, g\right)^{t}$, so this is what we wanted to show.

\section{Proof of the Main Theorem}

For the proof of the Main Theorem, we do not need the subgroup $X$ in the following lemma, but we want it for the applications in Section 8.

Lemma 7.1. Let $\Gamma, A$, and $X$ be Lie subgroups of a connected solvable Lie group $G$. Assume $A$ and $X$ are connected, and $A \subset X$. Then there are

- a finite-index subgroup $\Gamma^{\prime}$ of $\Gamma$;

- a maximal compact torus $T_{\text {cpct }}$ of $\overline{\operatorname{Ad} G}$;

- almost-complementary subtori $S$ and $S^{\perp}$ of $T_{\mathrm{cpct}}$; and

- an element $g_{0}$ of $G$;

such that

1. $\overline{\operatorname{Ad}_{G} \Gamma^{\prime}}$ is connected;

2. $S^{\perp}=\overline{\operatorname{Ad}_{G} \Gamma^{\prime}} \cap T_{\mathrm{cpct}}$ is a maximal compact torus of $\overline{\operatorname{Ad}_{G} \Gamma^{\prime}}$;

3. $\left(\overline{\operatorname{Ad}_{G} A^{g_{0}}} \cap S\right)\left(\overline{\operatorname{Ad}_{G} A^{g_{0}}} \cap S^{\perp}\right)=\overline{\operatorname{Ad}_{G} A^{g_{0}}} \cap T_{\text {cpct }}$ is a maximal compact torus of $\overline{\operatorname{Ad}_{G} A^{g_{0}}}$; and

4. $\left(\overline{\operatorname{Ad}_{G} X^{g_{0}}} \cap S\right)\left(\overline{\operatorname{Ad}_{G} X^{g_{0}}} \cap S^{\perp}\right)=\overline{\operatorname{Ad}_{G} X^{g_{0}}} \cap T_{\text {cpct }}$ is a maximal compact torus of $\overline{\operatorname{Ad}_{G} X^{g_{0}}}$.

Proof. We know $\overline{\operatorname{Ad}_{G} \Gamma}$ has only finitely many components (see 4.15), so there is a finite-index subgroup $\Gamma^{\prime}$ of $\Gamma$, such that $\overline{\operatorname{Ad}_{G} \Gamma^{\prime}}$ is connected.

Let $T_{\text {cpct }}$ be a maximal compact torus of $\overline{\operatorname{Ad} G}$, containing a maximal compact torus $S^{\perp}$ of $\overline{\operatorname{Ad}_{G} \Gamma}$. Let $T_{X}$ be a maximal compact torus of $\overline{\operatorname{Ad}_{G} X}$ that contains a maximal compact torus $T_{A}$ of $\overline{\operatorname{Ad}_{G} A}$. There is some $g_{0} \in G$ such that $T_{\text {cpct }}$ contains $T_{X}^{g_{0}}$ (see 4.22). For simplicity, we may replace $A$ and $X$ with $A^{g_{0}}$ and $X^{g_{0}}$, so we may assume $g_{0}=e$.

Let $S_{A}^{\perp}$ be the identity component of $\overline{\operatorname{Ad}_{G} A} \cap S^{\perp}$, and let $S_{X}^{\perp}$ be the identity component of $\overline{\operatorname{Ad}_{G} X} \cap S^{\perp}$. Let $S_{A}$ be a subtorus of $\overline{\operatorname{Ad}_{G} A}$ complementary to $S_{A}^{\perp}$ 
in $T_{A}$. Then $S_{A} \cap S^{\perp}$ is finite, so there is a subtorus $S_{X}$ of $T_{X}$ that contains $S_{A}$ and is almost complementary to $S_{X}^{\perp}$ in $T_{X}$. Then $S_{X} \cap S^{\perp}$ is finite, so there is a subtorus $S$ of $T_{\text {cpct }}$ that contains $S_{X}$ and is almost complementary to $S^{\perp}$.

The statement of the following theorem does not assume $G$ acts faithfully, because it may be necessary to pass to a finite cover in order to define the homomorphism $\tilde{\pi}$ for the nilshadow construction.

Main Theorem 7.2. Let $\Gamma$ be a properly discontinuous subgroup of a connected, solvable Lie group $G$ that acts transitively and almost faithfully on $\mathbb{R}^{n}$, such that $\mathbb{R}^{n} / \Gamma$ is compact. Let $A=G_{x_{0}}$ be the stabilizer of some point $x_{0} \in \mathbb{R}^{n}$ in $G$, let $X$ be a connected Lie subgroup of $G$ containing $A$, and let $\Gamma^{\prime}, T_{\text {cpct }}, S, S^{\perp}$, and $g_{0}$ be as in the conclusion of Lemma \%.1. Assume the natural homomorphism $\pi: G \rightarrow T_{\text {cpct }}$ lifts to a homomorphism $\tilde{\pi}: G \rightarrow \tilde{T}_{\text {cpct }}$, where $\tilde{T}_{\text {cpct }}=S \times S^{\perp}$, and let

$$
\Delta: G \rightarrow S \ltimes G: g \mapsto\left(g^{\sigma}, g\right)
$$

be the corresponding nilshadow map (see Definition 5.1). Then some finite-index subgroup $\Gamma^{\prime \prime}$ of $\Gamma^{\prime}$ has a syndetic hull $B^{\Delta}$ in $G^{\Delta}$, such that the crossed affine map

$$
\phi: B^{\Delta} \stackrel{\left.\Delta^{-1}\right|_{B} \Delta}{\longrightarrow} G \stackrel{g \mapsto x_{0} g_{0} g}{\longrightarrow} \mathbb{R}^{n}
$$

is a $\Gamma^{\prime \prime}$-equivariant diffeomorphism from $B^{\Delta}$ to $\mathbb{R}^{n}$. Therefore, $\phi$ induces a crossed affine map

$$
\phi^{\prime}: B^{\Delta} / \Gamma^{\prime \prime} \rightarrow \mathbb{R}^{n} / \Gamma^{\prime \prime}
$$

which is also a diffeomorphism.

Proof. By replacing $x_{0}$ with $x_{0} g_{0}$, we may assume $g_{0}=e$. Then, from Lemmas 7.1(3) and 5.10, we know $A^{\Delta}$ is a subgroup of $G^{\Delta}$. From Lemmas 7.1(2) and 5.10, we have $\left(\Gamma^{\prime}\right)^{\pi} \subset S^{\perp}$; so, from the definition of $\sigma$, we know $\left(\Gamma^{\prime}\right)^{\sigma} \subset S \cap S^{\perp}$, so $\left(\Gamma^{\prime}\right)^{\sigma}$ is finite. Therefore, replacing $\Gamma$ by a subgroup of finite index, we may assume $\Gamma^{\sigma}=e$. Then we may identify $\Gamma^{\Delta}$ with $\Gamma$. From $7.1(2)$, we know that $\overline{\operatorname{Ad}_{G} \Gamma}$ contains $S^{\perp}$. Therefore, Proposition 5.9 implies that $\overline{\operatorname{Ad}_{G^{\Delta}} \Gamma}$ contains a maximal compact torus of $\overline{\operatorname{Ad~} G^{\Delta}}$, so Proposition 4.25 implies that (some finite-index subgroup of) $\Gamma$ has a syndetic hull $B^{\Delta}$ in $G^{\Delta}$, such that $\left[B^{\Delta}, B^{\Delta}\right]$ contains every compact subgroup of $B^{\Delta}$. Because $\Gamma^{\sigma}=e$, the crossed affine map $\phi$ is $\Gamma$-equivariant, so it only remains to show that $\phi$ is a diffeomorphism.

We let

$$
B=\Delta^{-1}\left(B^{\Delta}\right)=\left\{b \in G \mid\left(b^{\sigma}, b\right) \in B^{\Delta}\right\},
$$

and define a map

$$
\rho: B \rightarrow A \backslash G: b \mapsto A b .
$$

(Warning: because $\Delta$ may not be a homomorphism, $B$ may not be a subgroup.) Since $A=G_{x_{0}}$, we know that $A \backslash G$ is $G$-equivariantly diffeomorphic to $\mathbb{R}^{n}$. Then, because $\phi$ is the composition

$$
\phi: B^{\Delta} \stackrel{\Delta^{-1}}{\longrightarrow} B \stackrel{\rho}{\longrightarrow} A \backslash G \stackrel{\approx}{\longrightarrow} \mathbb{R}^{n},
$$

it suffices to show that $\rho$ is a diffeomorphism.

Step 1. $A^{\sigma}$ normalizes $A$, so $A^{\Delta}$ is a subgroup of $G^{\Delta}$. This follows from Lemmas 7.1(3) and 5.10. 
Step 2. For every $s \in S$, we have $\left(A^{\Delta}\right)^{s} B^{\Delta}=G^{\Delta}$, and the intersection $\left(A^{\Delta}\right)^{s} \cap$ $B^{\Delta}$ is a maximal compact subgroup of $B^{\Delta}$. First, note that $\left(A^{\Delta}\right)^{s} \backslash G^{\Delta} / B^{\Delta}$ is compact, because $\left(A^{\Delta}\right)^{s} \backslash G^{\Delta} / \Gamma$ is compact (see 5.15) and $\Gamma \subset B^{\Delta}$. Second, note that $B^{\Delta}$ is proper on $\left(A^{\Delta}\right)^{s} \backslash G^{\Delta}$, because $\Gamma$ is proper on $\left(A^{\Delta}\right)^{s} \backslash G^{\Delta}$ (see 5.16) and $\Gamma$ is cocompact in $B^{\Delta}$.

Therefore, from Corollary $6.2^{\prime}$, we know that $\left(A^{\Delta}\right)^{s} B^{\Delta}=G^{\Delta}$. Then, since $\left(A^{\Delta}\right)^{s} \backslash G^{\Delta}$ is simply connected (see 5.13), we know that $\left(\left(A^{\Delta}\right)^{s} \cap B^{\Delta}\right) \backslash B^{\Delta}$ is simply connected (see 2.12), so $\left(A^{\Delta}\right)^{s} \cap B^{\Delta}$ contains a maximal compact subgroup of $B^{\Delta}$ (see 2.11). On the other hand, we know that $\left(A^{\Delta}\right)^{s} \cap B^{\Delta}$ is compact, because $B^{\Delta}$ acts properly. Therefore, $\left(A^{\Delta}\right)^{s} \cap B^{\Delta}$ is precisely a maximal compact subgroup of $B^{\Delta}$.

Step 3. $B^{\Delta}$ is simply connected and, for every $s \in S$, we have $\left(A^{\Delta}\right)^{s} \cap B^{\Delta}=e$. Since $A^{\Delta} \cap B^{\Delta}$ is compact (see Step 1 ), and $\left[B^{\Delta}, B^{\Delta}\right]$ contains every compact subgroup of $B^{\Delta}$, we have $A^{\Delta} \cap B^{\Delta} \subset\left[B^{\Delta}, B^{\Delta}\right]$. Therefore

$$
A^{\Delta} \cap B^{\Delta} \subset\left[B^{\Delta}, B^{\Delta}\right] \subset\left[G^{\Delta}, G^{\Delta}\right] \subset \operatorname{nil} G
$$

(see 5.17 and 2.14). Hence, $A^{\Delta} \cap B^{\Delta}$ is a normal subgroup of $G$ (see 2.10). Since $A^{\Delta} \cap G \subset A$, we also know that $A^{\Delta} \cap B^{\Delta} \subset A$. Then, since $A \backslash G$ is almost faithful, this implies $A^{\Delta} \cap B^{\Delta}$ is finite. However, from Step 2, we know $A^{\Delta} \cap B^{\Delta}$ is a maximal compact subgroup of $B^{\Delta}$, so it is connected (see 2.6). Hence $A^{\Delta} \cap B^{\Delta}$ is trivial.

From Step 2 and the preceding paragraph, we know that some maximal compact subgroup of $B^{\Delta}$ is trivial. Hence $B^{\Delta}$ is simply connected (see 2.6). Furthermore, it must be the case that every maximal compact subgroup of $B^{\Delta}$ is trivial (see 2.5), so, from Step 2, we see that, for every $s \in S$, we have $\left(A^{\Delta}\right)^{s} \cap B^{\Delta}=e$, as desired.

Step 4. The map $\rho$ is surjective. We wish to show $A B=G$. (This is more complicated than the proof of Lemma 5.11, because $B$ may not be a subgroup and we do not know whether $S$ normalizes $A$.) We know from Step 2 that $A^{\Delta} B^{\Delta}=G^{\Delta}$. Therefore, given any $g \in G$, there exist $a \in A$ and $b \in B$ such that

$$
\left(g^{\sigma}, g\right)=\left(a^{\sigma}, a\right) \cdot\left(b^{\sigma}, b\right)=\left((a b)^{\sigma}, a^{b^{\sigma}} b\right),
$$

so $g=a^{b^{\sigma}} b$ and $g^{\sigma}=(a b)^{\sigma}$.

Assume, for the moment, that $g \in \operatorname{ker} \sigma$. Then $(a b)^{\sigma}=g^{\sigma}=e$, so $b^{\sigma}=\left(a^{\sigma}\right)^{-1} \in$ $A^{\sigma}$, so $b^{\sigma}$ normalizes $A$, which means $a^{b^{\sigma}} \in A$. Therefore, $g=a^{b^{\sigma}} b \in A B$. Since $g \in \operatorname{ker} \sigma$ is arbitrary, we conclude that $\operatorname{ker} \sigma \subset A B$. Indeed, because $A^{\Delta}\left(B^{\Delta}\right)^{s}=$ $G^{\Delta}$ for every $s \in S$ (cf. Step 2), the same proof shows that $\operatorname{ker} \sigma \subset A B^{s}$, for every $s \in S$.

For an arbitrary $g \in G$, we have $g=a^{b^{\sigma}} b$. Since $\left(a^{b^{\sigma}}\right)^{\sigma}=a^{\sigma}$ (see 5.4), we have $a^{b^{\sigma}} \in a(\operatorname{ker} \sigma)$, so this implies $g \in a(\operatorname{ker} \sigma) b$. From the previous paragraph, we know $\operatorname{ker} \sigma \subset A B^{b^{\sigma}}$, so there is some $a_{1} \in A$ and some $b_{1} \in B$ such that $g=a\left(a_{1} b_{1}^{b^{\sigma}}\right) b$. We have $a a_{1} \in A$, because $A$ is a subgroup. We have

$$
\left(b_{1}^{b^{\sigma}} b\right)^{\Delta}=\left(b_{1}^{\sigma} b^{\sigma}, b_{1}^{b^{\sigma}} b\right)=\left(b_{1}^{\sigma}, b_{1}\right)\left(b^{\sigma}, b\right) \in B^{\Delta},
$$

because $B^{\Delta}$ is a subgroup, so $b_{1}^{b^{\sigma}} b \in B$. Hence $g \in A B$, as desired.

Step 5. The map $\rho$ is injective. Suppose $\rho(b)=\rho(c)$, with $b, c \in B$. This means $A b=A c$, so there is some $a \in A$ with $b=a c$. Then

$$
\left(b^{\sigma}, b\right)=\left((a c)^{\sigma}, a c\right)=\left(a^{\sigma}, a^{\left(c^{\sigma}\right)^{-1}}\right)\left(c^{\sigma}, c\right) \in\left(A^{\Delta}\right)^{\left(c^{\sigma}\right)^{-1}}\left(c^{\sigma}, c\right) .
$$


Since $\left(b^{\sigma}, b\right),\left(c^{\sigma}, c\right) \in B^{\Delta}$, then

$$
\left(b^{\sigma}, b\right)\left(c^{\sigma}, c\right)^{-1} \in\left(A^{\Delta}\right)^{\left(c^{\sigma}\right)^{-1}} \cap B^{\Delta}=e,
$$

from Step 3. Therefore, $b^{\Delta}=c^{\Delta}$, so $b=c$, as desired.

Step 6. The inverse of $\rho$ is $C^{\infty}$, so $\rho$ is a diffeomorphism. It suffices to show that $\rho$ is a submersion (see 4.31). Thus, it suffices to show $B$ is transverse to $A b$, for every $b \in B$. Because $\Delta: G \rightarrow G^{\Delta}$ is a diffeomorphism, it suffices to show $B^{\Delta}$ is transverse to $(A b)^{\Delta}$. We have

$$
\begin{aligned}
(A b)^{\Delta} & =\left\{\left((a b)^{\sigma}, a b\right) \mid a \in A\right\}=\left\{\left(a^{\sigma}, a^{\left(b^{\sigma}\right)^{-1}}\right)\left(b^{\sigma}, b\right) \mid a \in A\right\} \\
& =\left(A^{\Delta}\right)^{\left(b^{\sigma}\right)^{-1}} \cdot\left(b^{\sigma}, b\right),
\end{aligned}
$$

and we have $B^{\Delta}=B^{\Delta} \cdot\left(b^{\sigma}, b\right)$. From Step 2, we know $\left(A^{\Delta}\right)^{\left(b^{\sigma}\right)^{-1}} \cdot B^{\Delta}=G^{\Delta}$, which implies $\left(A^{\Delta}\right)^{\left(b^{\sigma}\right)^{-1}}$ is transverse to $B^{\Delta}$, because these are subgroups. Hence $(A b)^{\Delta}$ is transverse to $B^{\Delta}$, as desired.

\section{Geometric Flows on Solvtessellations}

Corollary 8.1. If a geometric flow on a solvtessellation has a dense orbit, then the flow is finitely covered by a solvflow.

Proof. Let $A \backslash G / \Gamma$ be a solvtessellation. In accordance with Proposition 2.4, let $X$ be a connected Lie subgroup of $G$ that contains $A$ as a codimension-one, normal subgroup. We wish to show there is a finite-index subgroup $\Gamma_{0}$ of $\Gamma$ and a connected Lie subgroup $X_{1}$ and a discrete subgroup $\Gamma_{1}$ of a connected, solvable Lie group $G_{1}$, such that the action of $X / A$ on $A \backslash G / \Gamma_{0}$ is $C^{\infty}$-isomorphic to the action of $X_{1}$ on $G_{1} / \Gamma_{1}$.

Let $\Gamma^{\prime}, T_{\text {cpct }}, S, S^{\perp}$, and $g_{0}$ be as in the conclusion of Lemma 7.1. Replacing $G$ by a finite cover, we may assume the natural homomorphism $\pi: G \rightarrow T_{\text {cpct }}$ lifts to a homomorphism $\tilde{\pi}: G \rightarrow \tilde{T}_{\text {cpct }}$, where $\tilde{T}_{\text {cpct }}=S \times S^{\perp}$. Let

$$
\Delta: G \rightarrow S \ltimes G: g \mapsto\left(g^{\sigma}, g\right)
$$

be the corresponding nilshadow map (see Definition 5.1).

Main Theorem 7.2 shows that some finite-index subgroup $\Gamma_{0}$ of $\Gamma^{\prime}$ has a syndetic hull $B^{\Delta}$ in $G^{\Delta}$, such that the crossed affine map

$$
\phi: B^{\Delta} \stackrel{\left.\Delta^{-1}\right|_{B} \Delta}{\longrightarrow} G \stackrel{g \mapsto A g_{0} g}{\longrightarrow} A \backslash G
$$

is a $\Gamma_{0}$-equivariant diffeomorphism from $B^{\Delta}$ to $A \backslash G$, and that $\phi$ induces a diffeomorphism $\phi^{\prime}: B^{\Delta} / \Gamma_{0} \rightarrow A \backslash G$.

Step 1. $S$ normalizes $A$ and centralizes $X / A$. Because $X$ has a dense orbit on $A \backslash G / \Gamma$, we know that $X g \Gamma$ is dense in $G$, for some $g \in G$. Applying $\sigma$, and then observing that $\overline{X^{\sigma}}$ is a subtorus of $S$, and $\Gamma^{\sigma}$ is finite, we conclude that $X^{\sigma}$ is dense in $S$. Since $X^{\sigma} \subset \overline{\operatorname{Ad}_{G} X}$ (see 5.10), this implies $S \subset \overline{\operatorname{Ad}_{G} X}$. Since $X$ normalizes $A$ and centralizes $X / A$, we know that $\overline{\operatorname{Ad}_{G} X}$ normalizes $A$ and centralizes $X / A$ (see 4.13 and 4.11 ), so we conclude that $S$ does likewise.

Step 2. Let $X_{1}=\phi^{-1}(A \backslash X)$. Then $X_{1}$ is a subgroup of $B^{\Delta}$, and the action of $X / A$ on $A \backslash G / \Gamma_{0}$ is $C^{\infty}$-isomorphic to the action of $X_{1}$ on $B^{\Delta} / \Gamma_{0}$. From Step 1 , we know $S$ normalizes $X$, so $X^{\Delta}$ is a subgroup of $G^{\Delta}$ (see 5.6). It is not difficult to see that $X_{1}=X^{\Delta} \cap B^{\Delta}$, so we conclude that $X_{1}$ is a subgroup of $B^{\Delta}$. 
For any $x^{\Delta} \in X_{1}$ and $b^{\Delta} \in B^{\Delta}$, we have

$$
\begin{aligned}
\left(x^{\Delta} b^{\Delta}\right)^{\phi} & =\left(x^{b^{-\sigma}} b\right)^{\Delta^{\phi}} & & (\Delta \text { is a crossed homomorphism) } \\
& =A x^{b^{-\sigma}} b & & (\text { definition of } \phi) \\
& =A x b & & \text { (Step 1) } \\
& =x A b & & (X \text { normalizes } A) \\
& =x\left(b^{\Delta}\right)^{\phi} & & \text { (definition of } \phi) .
\end{aligned}
$$

Therefore, $\phi$ is an isomorphism from the action of $X_{1}$ on $B^{\Delta}$ to the action of $X / A$ on $A \backslash G$, so $\phi^{\prime}$ is an isomorphism from the action of $X_{1}$ on $B^{\Delta} / \Gamma_{0}$ to the action of $X / A$ on $A \backslash G / \Gamma_{0}$.

Corollary 8.2. If a geometric flow on a solvtessellation has no nonconstant, $C^{\infty}$, invariant functions, then the flow is ergodic, and is finitely covered by an ergodic solvflow.

Proof. In the notation of the proof of Corollary 8.1, if $X^{\sigma}$ is not dense in $S$, then there are many nonconstant, $C^{\infty}$ functions on $S / \overline{X^{\sigma}} \Gamma^{\sigma}$. By precomposing with $\sigma$, these yield nonconstant, $C^{\infty}$, invariant functions on $A \backslash G / \Gamma$, contradicting our hypothesis. Therefore, it must be the case that $X^{\sigma}$ is dense in $S$. Then the proof of Corollary 8.1 shows that the geometric flow is finitely covered by some solvflow. Because quotients of ergodic flows are ergodic, it suffices to show that the solvflow is ergodic.

Suppose that the solvflow is not ergodic. Then it has a nonconstant, $C^{\infty}$, invariant function $f$ [BM, Thm. 6.1]. By averaging $f$ on the fibers of the projection to $A \backslash G / \Gamma$, we obtain a $C^{\infty}$, invariant function for the geometric flow. This average may be a constant function, but, by composing $f$ with a generic diffeomorphism of $\mathbb{R}$ before averaging, we can obtain a nonconstant average. This is a contradiction.

Corollary 8.3. Every $L^{2}$ eigenfunction of any ergodic geometric flow on any solvtessellation is equal to a $C^{\infty}$ function (a.e.).

Proof. From Corollary 8.2, we may assume the flow is a solvflow. Then every eigenfunction factors through to an eigenfunction of the maximal Euclidean quotient [BM, Thm. 6.2], which is isomorphic to a Kronecker flow [BM, Thm. 5.2]. The eigenfunctions of a Kronecker flow are the group characters, which are $C^{\infty}$.

Corollary 8.4. A geometric flow on a solvtessellation $\mathbb{R}^{n} / \Gamma$ is minimal (that is, every orbit is dense) if and only if (1) the flow has a dense orbit, and (2) the fundamental group $\Gamma$ is virtually nilpotent.

Proof. From Corollary 8.1, we know that the geometric flow on some finite cover $\mathbb{R}^{n} / \Gamma_{1}$ of $\mathbb{R}^{n} / \Gamma$ is isomorphic to a solvflow on some solvmanifold $G / \Gamma_{2}$.

$(\Rightarrow)$ Because the flow on $\mathbb{R}^{n} / \Gamma$ is minimal, it is clear that no orbit closure in $\mathbb{R}^{n} / \Gamma_{1}$ is contained in a compact subset of dimension less than $n$, so the same is true of orbits in $G / \Gamma_{2}$. Therefore, the proof of [A2, Thm. $\left.7 \mathrm{C}\right]$ shows that $\Gamma_{2}$ is nilpotent. Since $\Gamma_{1} \cong \Gamma_{2}$, we conclude that $\Gamma_{1}$ is nilpotent.

$(\Leftarrow)$ From Corollary 8.2, we know that the solvflow on $G / \Gamma_{2}$ is ergodic, so the solvflow is in general position. Furthermore, we may assume $\Gamma_{1}$ is nilpotent, which implies that $G / \Gamma_{1}$ is homeomorphic to a nilmanifold, so $G$ is class R [A2, Thm. 3.6]. Therefore, the solvflow is isomorphic to a nilflow [A2, Thm. 4.1], so it is minimal $[\mathrm{A} 2, \S 2]$. 


\section{REFERENCES}

[A1] L. Auslander, An exposition of the structure of solvmanifolds. Part I: Algebraic theory, Bull. Amer. Math. Soc. 79 (1973) 227-261. MR 58:6066a

[A2] L. Auslander, An exposition of the structure of solvmanifolds. Part II: $G$-induced flows, Bull. Amer. Math. Soc. 79 (1973) 262-285. MR 58:6066b

[AGH] L. Auslander, L. Green, and F. Hahn, Flows on Homogeneous Spaces, Princeton U. Press, Princeton, 1963. MR 29:4841

[AT] L. Auslander and R. Tolimieri, Splitting theorems and the structure of solvmanifolds, Ann. Math. 92 (1970) 164-173. MR 43:2733

[Ba] W. Baily, Introductory Lectures on Automorphic Forms, Princeton U. Press, Princeton, 1973. MR 51:2182

[B1] A. Borel, Density properties for certain subgroups of semi-simple groups without compact components, Ann. Math. 72 (1960) 179-188. MR 23:A964

[B2] A. Borel, Linear Algebraic Groups, 2nd ed., Springer-Verlag, New York, 1991. MR 92d:20001

[Bou] N. Bourbaki, Lie Groups and Lie Algebras, Part I, Addison-Wesley, Reading, Massachusetts, 1975. MR 89:1700 (reprint)

[BM] J. Brezin and C. C. Moore, Flows on homogeneous spaces: a new look, Amer. J. Math. 103 (1981) 571-613. MR 83e:22009

[CE] H. Cartan and S. Eilenberg, Homological Algebra, Princeton, Princeton, 1956. MR 17:1040e

[C] L. S. Charlap, Bieberbach Groups and Flat Manifolds, Springer-Verlag, New York, 1986. MR 88j: 57042

[CW] P. B. Chen and T. S. Wu, On full subgroups of solvable groups, Amer. J. Math. 108 (1986) 1487-1506. MR 88c:22010

[Da] S. G. Dani, On ergodic quasi-invariant measures of group automorphism, Israel J. Math. 43 (1982) 62-74. MR 85d:22017

[D] J. Dieudonné, Foundations of Modern Analysis, Academic Press, New York, 1960. MR 22:11074

[FJ] F. T. Farrell and L. E. Jones, Classical Aspherical Manifolds (CBMS \#75), Amer. Math. Soc., Providence, 1990. MR 91k:57001

[FG] D. Fried and W. Goldman, Three-dimensional affine crystallographic groups, Adv. Math. 47 (1983) 1-49. MR 84d:20047

[Ha] M. Hall, Jr., The Theory of Groups, Macmillan, New York, 1959. MR 21:1996

[Ho] G. Hochschild, The Structure of Lie Groups, Holden-Day, San Francisco, 1965. MR 34:1796

[Hu] J. E. Humphreys, Linear Algebraic Groups, Springer-Verlag, New York, 1975. MR 53:633

[I1] K. Iwasawa, On some types of topological groups, Ann. Math. 50 (1949) 507-558. MR 10:679a

[I2] K. Iwasawa, Über nilpotente topologische Gruppen, Proc. Japan Acad. 21 (1945) (1949) 124-137. MR 11:325e

[M] J. M. Montesinos, Classical Tessellations and Three-Manifolds, Springer-Verlag, New York, 1987. MR 89d:57016

[M1] G. D. Mostow, Factor spaces of solvable groups, Ann. Math. 60 (1954) 1-27; 66 (1957), 590. MR 15:853g; MR 19:752d

[M2] G. D. Mostow, On the fundamental group of a homogeneous space, Ann. Math. 66 (1957) 249-255. MR 19:561e

[P] W. Parry, Dynamical representations in nilmanifolds, Compositio Math. 26 (1973) 159174. MR 47:8816

[R] M. S. Raghunathan, Discrete Subgroups of Lie Groups, Springer-Verlag, New York, 1972. MR 58:22394a

[V] V. S. Varadarajan, Lie Groups, Lie Algebras, and Their Representations, SpringerVerlag, New York, 1984. MR 85e:22001

[Wa] C. T. C. Wall, Surgery on Compact Manifolds, Academic, London/New York, 1970. MR 55:4217

[W1] D. Witte, Zero-entropy affine maps on homogeneous spaces, Amer. J. Math. 109 (1987) 927-961; 118 (1996), 1137-1140. MR 88i:28038; MR 97j:28043 
[W2] D. Witte, Supperrigidity of lattices in solvable Lie groups, Invent. Math. 122 (1995) 147-193. MR 96k:22024

[Z] R. J. Zimmer, Ergodic Theory and Semisimple Groups, Birkhäuser, Boston, 1984. MR 86j:20014

Department of Mathematics, Williams College, Williamstown, MA 01267

Current address: Department of Mathematics, Oklahoma State University, Stillwater, Oklahoma 74078

E-mail address: dwitte@math.okstate.edu 